\title{
Production et consommation de céramiques à Potamia (Chypre) de
} l'époque franque à l'époque ottomane

Véronique François, Lucy Vallauri

\section{Citer ce document / Cite this document :}

François Véronique, Vallauri Lucy. Production et consommation de céramiques à Potamia (Chypre) de l'époque franque à l'époque ottomane. In: Bulletin de correspondance hellénique. Volume 125, livraison 2, 2001. pp. 523-546;

doi : 10.3406/bch.2001.7151

http://www.persee.fr/doc/bch_0007-4217_2001_num_125_2_7151

Document généré le 19/05/2016 


\begin{abstract}
In the context of the project "Potamia-Hagios Sozomenos, the constitution of the countryside in the médiéval East" the pottery collected during the course of the récent survey hâve, in spite of its fragmentary condition, provided fresh information at the site level, but also for the island as a whole, in the Frankish and Ottoman periods. Study of the pottery is informative both for the manufacture of local and régional Cypriot pottery and for the dissémination of pottery originating in the Western and Eastern Mediterranean basins. The fragmentary condition of the material is offset by its large volume and the récurrent character of the différent catégories of pottery, identified by types and chronological periods. This article proposes a new considération of Cypriot ceramic typol- ogy in the light of previous research and récent discoveries.
\end{abstract}

\title{
Résumé
}

Dans le cadre du programme de recherche "Potamia-Hagios Sozomenos, la constitution des paysages dans l'Orient médiéval ", les céramiques recueillies au cours de la récente prospection ont, malgré leur état très fragmentaire, livré de nouvelles informations à l'échelle du site mais aussi à l'échelle de l'île pour les époques franque et ottomane. Leur étude renseigne à la fois sur la fabrication de céramiques chypriotes locales ou régionales et sur la circulation de vaisselles originaires des bassins occidental et oriental de la Méditerranée. L'état fragmentaire du matériel est compensé par son volume important et par l'aspect répétitif des différentes catégories de céramiques, identifiées par types et par périodes chronologiques. Cet article propose un nouveau bilan du faciès céramologique chypriote au vu des recherches antérieures et des découvertes récentes.

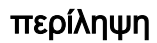

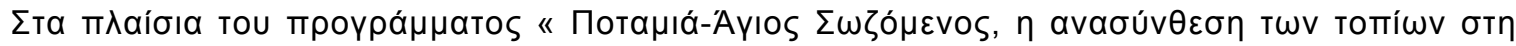

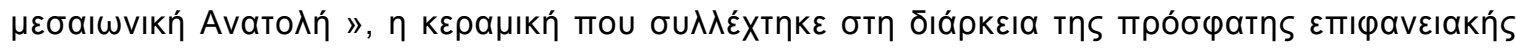

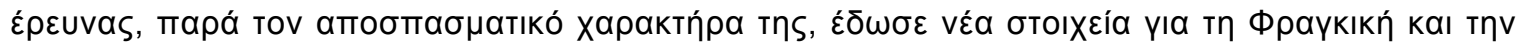

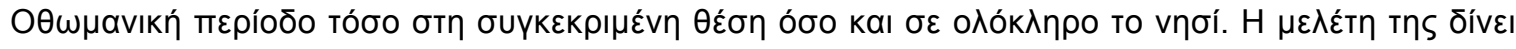

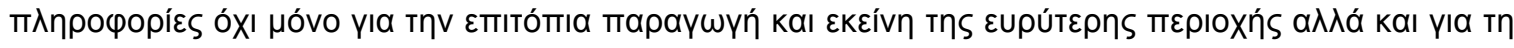

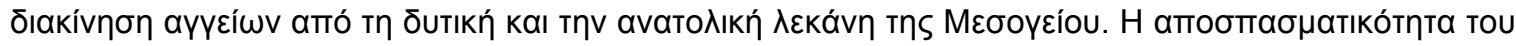

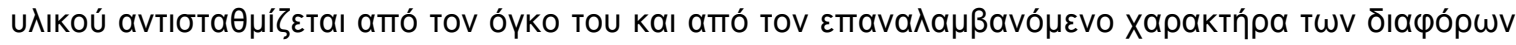

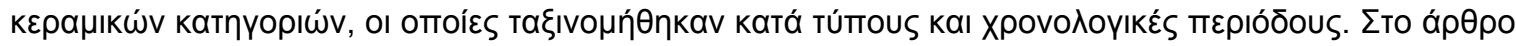

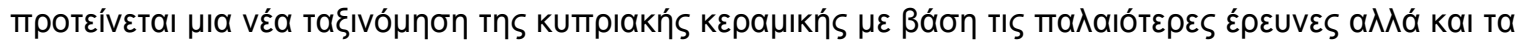

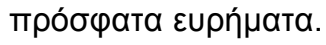




\section{Production et consommation de céramiques à Potamia (Chypre) de l'époque franque à l'époque ottomane}

par Véronique FRANÇOIS et LUCY VALLAURI*

Dans le cadre du programme de recherche "Potamia-Agios Sozomenos. La constitution des paysages dans l'Orient médiéval ", l'étude du matériel recueilli lors de la prospection de juillet $2000^{1}$ à Potamia (fig. 1) a livré de nouvelles données sur la fabrication de céramiques chypriotes locales ou régionales et sur la circulation de vaisselles originaires des bassins occidental et oriental de la Méditerranée. L'état fragmentaire de la documentation est compensé par le volume important des découvertes et l'aspect répétitif des différentes catégories de céramiques, identifiées par types et par périodes chronologiques. Cet article propose un nouveau bilan du faciès céramologique chypriote au vu des données antérieures, qui ne sont pas négligeables, et des découvertes récentes.

\section{Rappel des données antérieures - fouilles et collections}

L'étude des céramiques médiévales à Chypre relève d'une longue tradition et s'est surtout portée sur la vaisselle fine à glaçure, tandis que la céramique commune était délaissée. Cependant nombre de publications - études céramologiques, rapports de fouille ou catalogues de collections - ont été consacrées aux productions chypriotes d'époque franque. Nous proposons d'en dresser ici un inventaire non exhaustif afin de replacer nos découvertes de Potamia dans un contexte local, régional puis national.

* CNRS, LAMM, MMSH, Aix-en-Provence.

1 Voir dans cette même livraison, N. LeCUYER et al., "Pota-

mia-Agios Sozomenos (Chypre). La constitution des paysages

dans l'Orient médiéval *, infra, p. 655-678. 


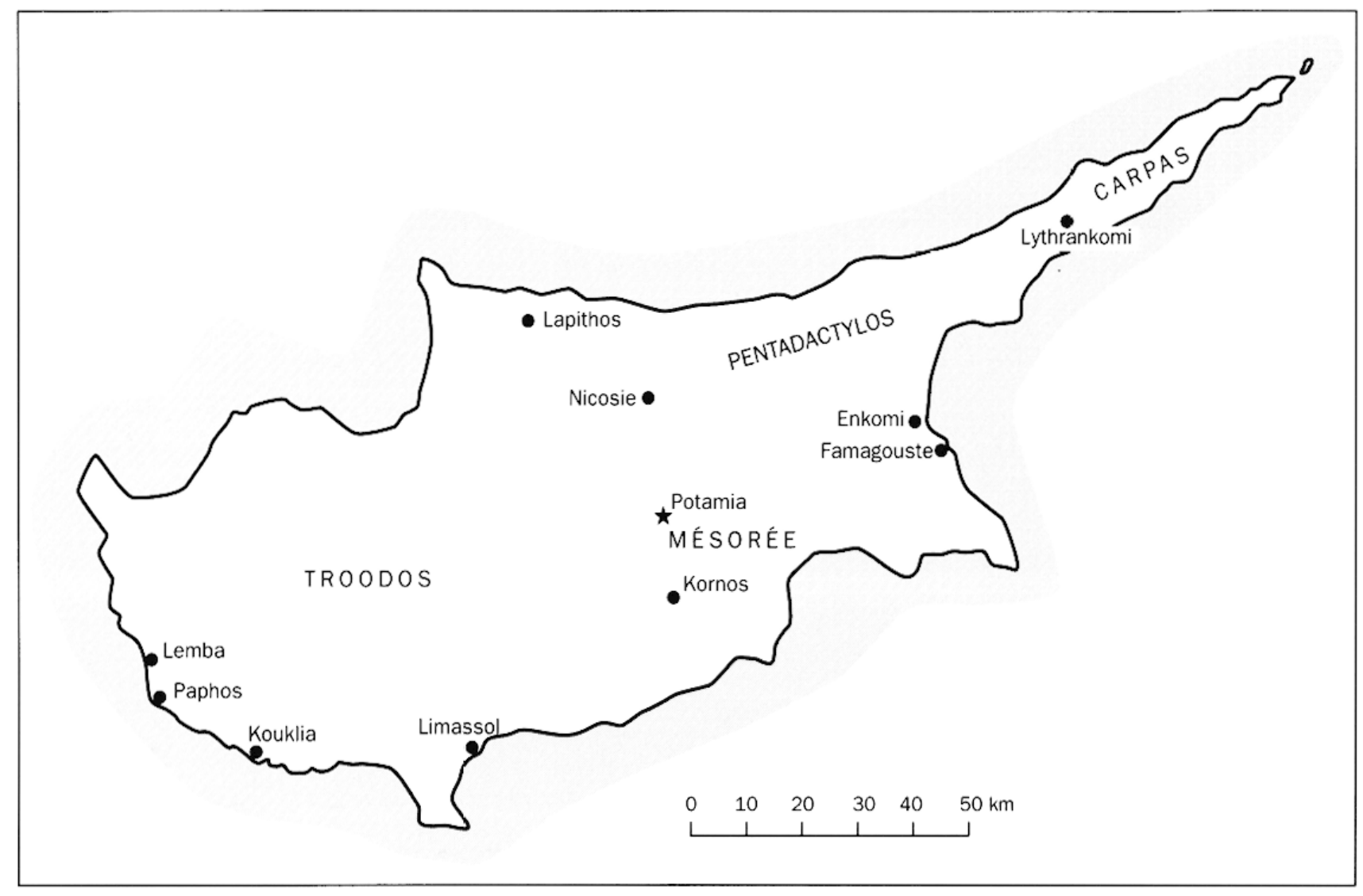

Fig. 1. Carte de Chypre avec les principaux lieux cités.

\section{A. Les données locales}

Une prospection ancienne et des travaux récents semblent avoir livré les indices d'une activité potière à Potamia à l'époque médiévale. En 1957, une équipe dirigée par $\mathrm{H}$. W. Catling investit la région de Potamia ${ }^{2}$. La prospection menée dans la vallée du Gialias conduit notamment à la découverte de cinquante-sept sites médiévaux et parmi eux, non loin du manoir royal des Lusignan, à Archangelos, un four de potier entouré de fragments de pots à sucre du type en usage dans les raffineries sucrières de Kolossi et Kouklia. Malheureusement, H. W. Catling n'en livre aucun dessin ni aucune photographie. Plus récemment, lors de travaux de voirie à Agios Sozomenos, B. J. Walker signale la mise au jour de tombes creusées entre les églises d'Agios Mamas et d'Agios Georgios ${ }^{3}$; à cette occasion, de nombreux fragments de céramique à glaçure ont été découverts ${ }^{4}$. Présentés comme une production locale, ils sont datés de la fin du XIVe-XVe siècle.

$2 \mathrm{H}$. W. CATLING, "The Ancient Topography of the Yalias Valley ", RDAC 1982, p. 227-236. Une partie du matériel recueilli par l'équipe de Catling est publiée par M.-L. VON WARTBURG, "Cane Sugar Production Sites in Cyprus. Real and Imagined ", RDAC 2000, p. 392-395, 398-400.
3 B. J. WALKER, The Ceramic Correlates of Decline in the Mamluk Sultanate: An Analysis of Late Medieval Sgraffito Wares, $\mathrm{Ph}$. Thesis, University of Toronto (1998), p. 133.

4 Ils sont conservés au CAARI à Nicosie mais cette information nous étant parvenue trop tard, il ne nous a pas été possible de voir ce matériel. 
Cependant, en l'absence d'indications supplémentaires et d'images lisibles, il est difficile de vérifier cette affirmation. Sans remettre en question la présence de vases dans les tombes - une pratique habituelle à Chypre - l'existence d'ateliers produisant de la céramique glaçurée à Potamia n'est pas prouvée, l'unique indice dont on dispose actuellement étant une pernette (trépied de terre cuite facilitant l'enfournement des vases à glaçure) trouvée lors de la prospection.

\section{B. Les données régionales}

Si Potamia ne peut être, en l'état actuel de nos connaissances, considérée avec certitude comme une zone de fabrication de céramique à glaçure, il n'en est pas de même de Nicosie, située à $25 \mathrm{~km}$ de là. En 1947, à l'occasion de fouilles de sauvetage à proximité de l'avenue Stassinos, une artère parallèle aux douves creusées au pied de la muraille vénitienne, A. H. S. Megaw découvre plusieurs fours de potier ${ }^{6}$. La céramique associée à ces structures n'appartiendrait pas, selon l'auteur, au groupe des céramiques fines sgraffito chypriotes du XIVe siècle mais plutôt à une production du XIII siècle. Diverses campagnes postérieures ont livré d'autres tessons médiévaux tardifs ${ }^{7}$, la découverte la plus récente étant celle d'un puits dégagé en 1988 à l'angle des rues Androcleous et Pindarou au Sud-Est de la ville médiévale, à l'intérieur de l'enceinte des Lusignan, et non loin des fours de A. H. S. Megaw. Ce puits appareillé contenait une série de vases particulièrement intéressants pour nous car une partie d'entre eux sont de mêmes types que les fragments de Potamia. Une monnaie de Hugues I ${ }^{e r}(1205-1218)$ trouvée au fond du puits laisse croire qu'il est contemporain des structures de cuisson voisines et fournit un terminus post quem. Le remplissage du puits contenait un bel ensemble de matériel associé: des vases à pâte claire ainsi que de la céramique culinaire à pâte rouge glaçurée, deux groupes identiques aux découvertes faites dans le quartier de Chrysaliniotissa à Nicosie et datées du XIVe siècle ${ }^{8}$; de la vaisselle de table peinte à l'engobe du XIII siècle, à glaçure monochrome, et de la vaisselle incisée et rehaussée de glaçures polychromes, datée du XIVe siècle, ainsi que quelques importations venues probablement de Syrie - une coupe à pâte siliceuse et décor peint en noir sous glaçure alcaline incolore du XIVe siècle, de même type que certains vases de Chrysaliniotissa', et un grand plat à pâte argileuse et glaçure plombifere sans doute issu des ateliers d'Al Mina en activité au XIII' siècle. L'existence probable de fours à Nicosie au XIII siècle et la présence répétée, dans la capitale, de vases très proches de - pour ne pas dire identiques à - certaines catégories mises en évidence par notre étude, nous conduisent à accorder une grande attention à ces trouvailles.

5 J. DU PLAT TAYLOR, * Medieval Graves in Cyprus *, Ars Islamica V (1938), p. 55-86; A. PAPAGEORGIOU, " "Epeuva ciç tòv vaòv

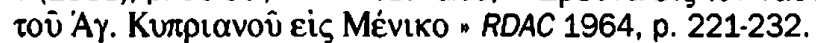

6 Information tirée du rapport de fouille rédigé par A. H. S. Megaw et rapportée par P. FloURENTZOS, A Hoard of Medieval Antiquities from Nicosia (1994), p. 2-3.
7 Ibid., p. 3 ; A. H. S. MEGAW, * Three Medieval Pit Groups from Nicosia ", RDAC 1937-1939 [1951], p. 145-168. 8 lbid., p. 150, fig. 7 A.25.

9 Ibid., p. 148. 


\section{Les données nationales}

C'est sans doute avec l'article de J. Du Plat Taylor consacré aux tombes médiévales d'Episkopi et à leur contenu que commence véritablement l'étude des céramiques médiévales et modernes trouvées dans l'île ${ }^{10}$. Il est suivi quelques années plus tard par une première classification établie à partir des principales découvertes archéologiques de vaisselles faites à Chypre et à partir de collections privées ${ }^{11}$. Cet intérêt pour la poterie augmente grâce aux fouilles de Polis et du château de Saranda Kolones à Paphos, qui livrent à la fois des importations byzantines et des productions chypriotes ${ }^{12}$. A. H. S. Megaw est le grand promoteur de cette recherche. À partir des années 1970, les fouilles de Kouklia (Palaipaphos) dirigées par F. Maier vont régulièrement mettre au jour de la vaisselle de table chypriore ainsi que des pots à mélasse et des formes à sucre. Les découvertes de ce site sont traitées depuis 1995 par M.-L. von Wartburg ${ }^{13}$. Les fouilles italiennes de Garrison's Camp à Paphos fournissent elles aussi, depuis le début des années 1990, du matériel médiéval et ottoman ${ }^{14}$. C'est à D. Papanikola-Bakirtzis que revient la mise en lumière des productions de plusieurs centres dispersés dans l'île, Enkomi, Paphos et Lapithos $^{15}$. En 1996, elle rédige un ouvrage de synthèse sur les ateliers chypriotes des XIII ${ }^{\mathrm{e}} \mathrm{XVI}{ }^{\mathrm{e}}$ siècles qui reste aujourd'hui le livre de référence ${ }^{16}$. Par ailleurs, les riches collections muséographiques et privées dispersées dans le monde proposent, à travers leurs catalogues, de très bonnes illustrations de cette vaisselle ${ }^{17}$.

Nous savons donc qu'il existe à Chypre, à l'époque du royaume des Lusignan (11921489), une tradition potière bien établie; plusieurs ateliers dispersés dans l'île en témoignent. Dans la région prospère de Paphos, du début du XIII ${ }^{e}$ au milieu du XIVe siècle, cet artisanat

10 J. Du PLAT TAYLOR, loc. cit. (supra, n. 5), p. 55-86.

11 J. DU PLAT TAYLOR, A. H. S. MEGAW, "Cypriot Medieval Glazed Pottery. Notes for a Preliminary Classification ", RDAC 1937-1939 [1951], p. 1-13.

12 A. H. S. MEGAW, A. DiKIGOROPOULOS, " Early Glazed Pottery from Polis ", RDAC 1940-1946 [1958], p. 77.93; A. H. S. MEGAW, "Excavations at Saranda Kolones, Paphos. Preliminary Report on the 1966-67 and 1970-71 Seasons ", RDAC 1971, p. 117146.

13 M.-L. VON WARTBURG, "Design and Technology of the Medieval Cane Sugar Refineries in Cyprus. A Case Study in Industrial Archaeology ", in A. MALPICA (éd.), Paisajes del azúcar, Actas del quinto seminario internacional sobre la caña de azúcar, Motril, 20-24 de septiembre de 1993 (1995), p. 83-116; ead., "Medieval Glazed Pottery from the Sanctuary of Aphrodite at Palaipaphos (Site TA). A Preliminary Survey ", in F. G. MAIER, M.-L. VON WARTBURG, "Excavations at Kouklia (Palaipaphos), Eighteenth Preliminary Report: Seasons 19931995 ", RDAC 1997, p. 184-194; ead., "Lemba Ware Reconsidered ", ibid., p. 323-340.

14 F. GIUDICE et al., "Paphos, Garrison's Camp, Campagna 1988 ", RDAC 1992, p. 205-250; id., "Paphos, Garrison's Camp. Campagna 1989 ", RDAC 1993, p. $279-327$; id., "Paphos, Garrison's Camp, Campagna 1990 ", RDAC 1994, p. 215-268; id., " Paphos, Garrison's Camp, Campagna
1991 ", RDAC 1996, p. 171-268.

15 D. PAPANIKOLA-BAKIRTZIS, "Medieval Pottery from Enkomi, Famagusta n, in V. DÉROCHE, J.-M. SPIESER (éds), Recherches sur la céramique byzantine, BCH Suppl. XVIII (1989), p. 233. 246; ead., "Cypriot Medieval Glazed Pottery: Answers and Questions ", in The Sweet Land of Cyprus, Papers Given at the 25th Jubilee Spring Symposium of Byzantine Studies, Birmingham, March 1991 (1993), p. 115-130.

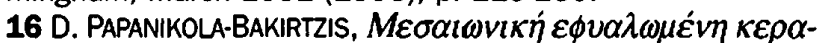

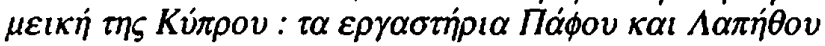
(1996).

17 A. Brown, H. Catulng, "Additions to the Cypriot Collection in the Ashmolean Museum, Oxford 1963-77 ", OAth 13 (1980), p. 124-137 ; D. PAPANIKOLA-BAKIRTZIS, Céramique chypriote médiévale au Musée de la Fondation Piéridès (1989); E. PILTZ, The von Post Collection of the Cypriote Late Byzantine Glazed Pottery, Studies in Mediterranean Archaeology

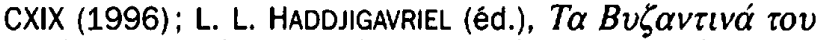

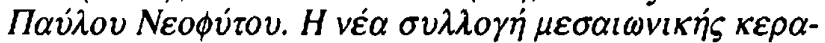

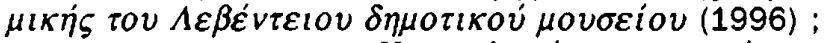
D. PAPANIKOLA-BAKIRTZIS, "H $\varepsilon \phi u \alpha \lambda \omega \mu \varepsilon \dot{v} \eta \kappa \varepsilon \rho \alpha \mu \mathrm{L} \kappa \dot{\eta} \sigma \tau \eta$

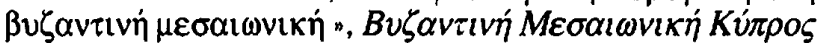

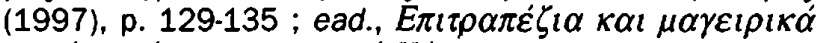

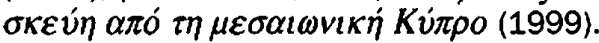


constitue un secteur d'activité important. Le centre d'Enkomi fonctionne à peu près à la même époque, autour de la fin XIII'-XIV"e siècle. Ce sont ensuite les officines de Lapithos qui prennent le relais et poursuivent leur activité jusqu'au XVIc siècle. D'autres découvertes de l'époque des Lusignan, faites sur des sites de consommation ou à l'intérieur de tombes, fournissent des exemples supplémentaires des productions chypriotes sans qu'il soit pour autant possible de les attribuer aux ateliers déjà identifiés. Grâce à toutes ces recherches, les caractéristiques typologiques des vases fabriqués dans l'île, c'est-à-dire la nature de leur pâte, leur forme, les techniques d'ornementation ainsi que leur décor, sont aujourd'hui assez bien connus.

\section{Méthode d'investigation}

Notre étude s'est concentrée sur les parcelles à plus forte densité de matériel et sur les secteurs qui présentaient une problématique particulière - zone de production potière, zone liée à l'éventuelle culture de la canne à sucre et installations hydrauliques. Dans ce cadre, 46000 tessons ont été examinés et n'ont été retenus pour élaborer notre classement que les éléments de forme, le revêtement et le décor. Ceci nous a conduit à rejeter $80 \%$ du matériel, un chiffre énorme qui inclut tous les fragments informes de l'Antiquité à l'époque ottomane. À partir de tessons " marqueurs ", c'est-à-dire identifiés avec sûreté, nous avons pu distinguer trois grandes séquences chronologiques et calculer les volumes par périodes: l'Antiquité et l'Antiquité tardive (du $\mathrm{VI}^{\mathrm{e}} \mathrm{s}$. av. au VII $\mathrm{e}$. ap. J.-C.) sont représentées par $19 \%$ du matériel; la période médiévale franque (du XIII cau début du XVIc s.) par $78 \%$ du matériel; l'époque ottomane (de la fin du $\mathrm{XVI}^{\mathrm{e}}$ au début du XIXe s.) par $3 \%$ du matériel. Pour les périodes qui nous intéressent 6237 échantillons ont été reconnus; ils constituent la base de notre recherche (tableau 1). Les différentes catégories identifiées sont d'une part les productions chypriotes, c'est-à-dire les céramiques communes sans revêtement en pâte claire et en pâte rouge, les céramiques glaçurées de cuisine et de table, la vaisselle fine engobée et glaçurée (tableau 2) et d'autre part les importations, qui incluent des céramiques glaçurées de cuisine et de table, des pâtes siliceuses et des faïences. Ces identifications témoignent de la grande diversité des poteries en usage dans l'île du XIV' au début du XIX siècle.

\begin{tabular}{cc|cc}
\hline \multicolumn{2}{c|}{ Époque franque } & \multicolumn{2}{c}{ Époque ottomane } \\
\hline Productions chypriotes $\quad$ Importations & Productions chypriotes & Importations \\
\hline 5931 tessons & 64 tessons & 62 tessons & 180 tessons \\
\hline
\end{tabular}

Tableau 1. Nombre de tessons identifiés par période et par origine. 


\begin{tabular}{ccccccc}
\hline $\begin{array}{c}\text { Céramique } \\
\text { commune } \\
\text { à pâte claire }\end{array}$ & $\begin{array}{c}\text { Céramique } \\
\text { commune } \\
\text { à pâte rouge }\end{array}$ & $\begin{array}{c}\text { Céramique } \\
\text { glaçurée }\end{array}$ & $\begin{array}{c}\text { Céramique } \\
\text { de Paphos } \\
\text { incisée }\end{array}$ & $\begin{array}{c}\text { Céramique } \\
\text { de Lapithos } \\
\text { incisée à } \\
\text { peinture } \\
\text { polychrome }\end{array}$ & $\begin{array}{c}\text { Céramique } \\
\text { de Lapithos } \\
\text { incisée à } \\
\text { peinture } \\
\text { monochrome }\end{array}$ & $\begin{array}{c}\text { Céramique } \\
\text { de Lapithos } \\
\text { peinte }\end{array}$ \\
\hline $32 \%$ & $31 \%$ & $17 \%$ & $1 \%$ & $12 \%$ & $2 \%$ & $5 \%$ \\
\hline
\end{tabular}

Tableau 2. Volume des productions médiévales chypriotes par catégorie.

\section{Vaisselle et céramiques en usage aux époques franque et ottomane}

\section{A. Période médiévale}

\section{A. 1. Vaisselle de cuisine et de stockage à pâte claire et à pâte rouge locale et régionale}

Pour l'époque médiévale, le faciès céramique mis en évidence à Potamia est essentiellement d'origine chypriote puisqu'on note seulement $1 \%$ d'importations. Les céramiques communes dominent (soit $63 \%$ en moyenne) et se répartissent de façon quasi égale entre des céramiques à pâte calcaire claire sans revêtement et des céramiques à pâte rouge grossière sans revêtement ou glaçurée.

L'argile calcaire fine et plastique pourrait correspondre aux marnes reconnues dans la composition géologique du site ${ }^{18}$ et plusieurs indices plaident en faveur d'une production locale. Sur vingt-deux parcelles cadastrales prospectées, situées de part et d'autre de l'Alykos, on note la présence de formes surcuites et déformées en tout point identiques à celles de vases bien conservés (fig. $2, \mathbf{n}^{\text {os }} 4,13$; fig. $\left.9, n^{\circ} 1\right)^{19}$. Ces " moutons " apparaissent sur les parcelles à forte densité de pâte calcaire mais leur aire de dispersion sur plusieurs zones pose néanmoins un problème pour la localisation du ou des ateliers, de dépotoirs et de fours pour la cuisson. L'absence de brique de four, ou d'artifice de potier dans les zones étudiées, pourrait signifier que les officines étaient implantées peut-être au pied de pente sous les falaises où les bancs d'argile affleurent, suivant des exemples fréquemment observés en Méditerranée ${ }^{20}$. Ces présomptions d'une production locale sont corroborées par les observations faites par H. W. Catling en 1957, qui signale des vestiges d'un four de potier médiéval à Archangelos et des fragments de pots à sucre. Cependant, ces indices ont aujourd'hui disparu, probablement submergés et emportés lors du déplacement du cours de la rivière.

18 Voir le rapport sur la géomorphologie et l'alluvionnement, infra, p. 662-667.

19 Tous les dessins sont des auteurs.
20 Exemples en Catalogne, Provence et Languedoc, H. AMOURIC, J. THIRIOT, J.-L. VAYSSETTES, "Ateliers en grotte: apport contrasté des sources écrites et des données de terrain ", in l jornadas de cerâmica medieval e pós-medieval, Tondela 1992 (1995), p. 263-269. 


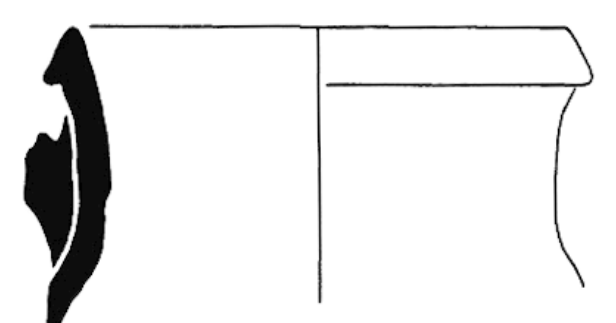

1

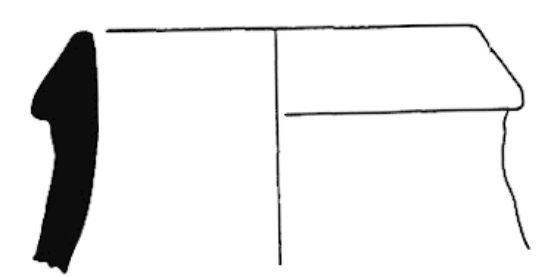

2

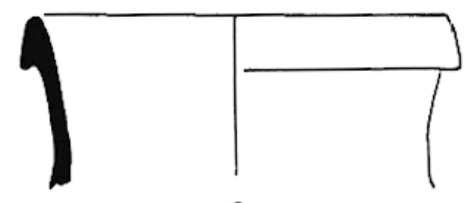

3
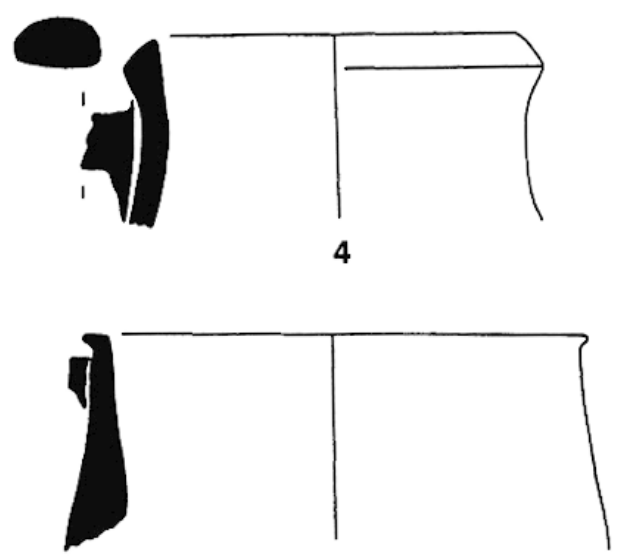

5
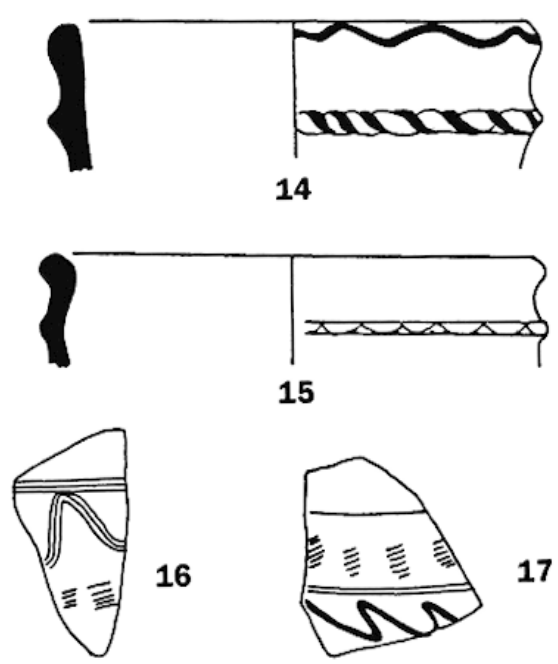
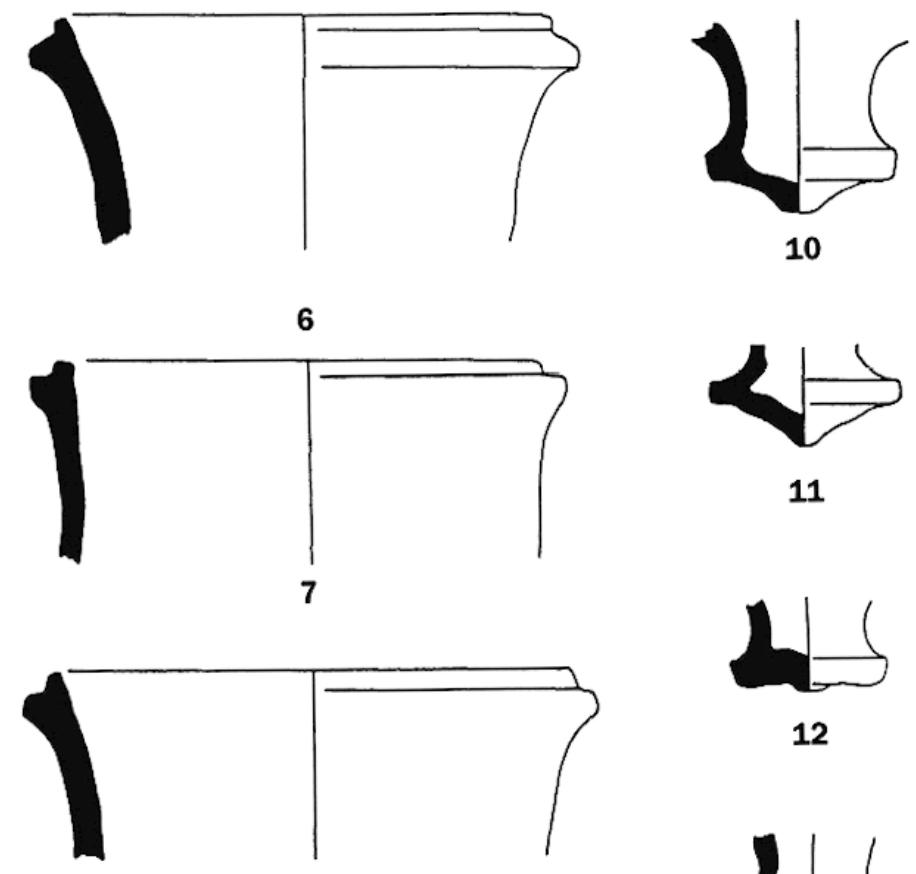

8

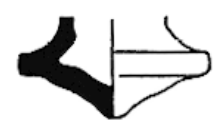

11

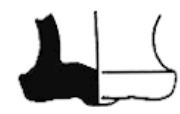

12

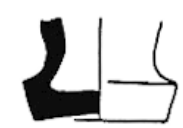

13

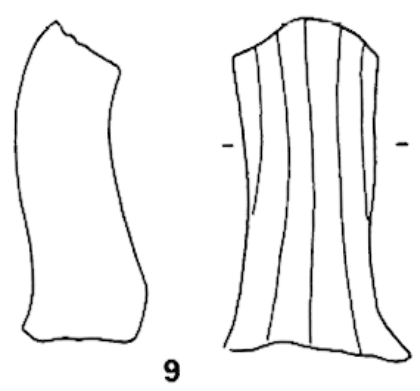

لـ
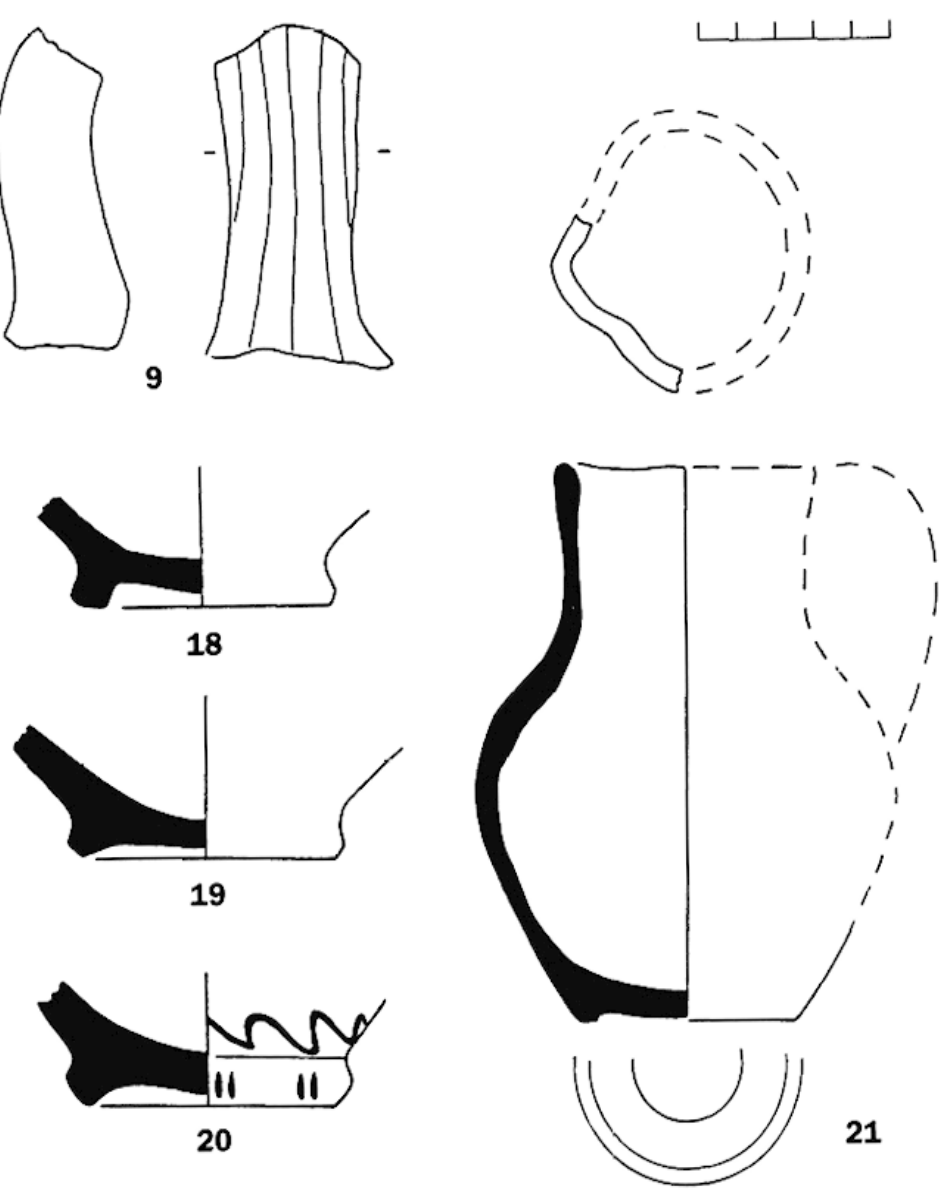

Fig. 2. Vaisselle de cuisine et de stockage à pâte calcaire claire. 
Les formes produites avec cette argile sont des cruches à col haut, fond quasi plat mais dégagé sous le pied, anse cannelée et lèvre en bandeau ou poulie (fig. $2, \mathrm{n}^{\text {os }} \mathbf{1 - 9}, 14-20$ ). Les différents fragments de cols montrent une certaine standardisation dans les dimensions $(12$ à $13 \mathrm{~cm}$ de diamètre). La production tournée reste assez grossière et l'épaisseur des parois a souvent été raclée à la lame. Cette production calcaire est comparable à celle qui a été retrouvée dans le puits à Nicosie, c'est-à-dire deux pichets à une anse, un gros bol, un vase brisé et une amphorette à deux anses et lèvre en bandeau qui correspond bien au type trouvé à Potamia ${ }^{21}$. L'usage de vases à liquide en pâte calcaire est néanmoins reconnu sur le site de Potamia, comme le montre le petit pot à bec pincé qui contenait le trésor exhumé à Agios Sozomenos, daté par les monnaies d'argent de la seconde moitié du XIVe siècle (fig. 2, $\mathrm{n}^{\circ} 21$ ). Quelques cordons rapportés et pincés ainsi que des décors d'ondes et de stries réalisés au peigne caractérisent cette production de cruches et de jattes (fig. 2, nos 14-17). Des godets de noria ont également été tournés dans cette argile. Cette forme à usage spécifique pour remonter l'eau est bien identifiée par les bords en poulie et les fonds en toupie qui permettaient de les attacher sur la roue (fig. $2, n^{\text {os }} 10-13$ ). Le modèle archaïque renvoie à celui attesté en Ifriqya, en Égypte et en Sicile dès le IX $\mathrm{X}^{\mathrm{e}}$ et le $\mathrm{X}^{\mathrm{c}}$ siècle mais dont la forme perdure encore dans la vallée du Nil22. Leur présence parmi les surcuits indique une production certaine et un usage intensif lié à la présence des norias sur le site ${ }^{23}$.

L'argile rouge, granuleuse et ferrugineuse, a été utilisée pour façonner des vases à usages divers. Elle pourrait avoir une origine régionale, comme l'atteste la longue tradition du travail de la terre à Kornos, situé à une vingtaine de kilomètres de Potamia ${ }^{24}$. Ce petit village a conservé une technique de fabrication entièrement montée à la main et réalisée par des femmes. Les céramiques sont modelées et la tournette y est employée pour les différentes phases de la finition (fig. $9, \mathrm{n}^{\circ} 2$ ). La terre grossière, capable de résister aux chocs thermiques, est employée pour les pots culinaires mais elle est peu adaptée à l'élaboration de la vaisselle de table. Par ailleurs, sa texture poreuse favorise l'évaporation et la conservation de l'eau fraîche ou des grains; c'est pourquoi elle convient aussi à l'élaboration des grosses jarres rondes de stockage inamovibles et des petits vases à liquide pour le transport ou pour le travail dans les champs. Dans la production actuelle, la glaçure n'est plus utilisée.

21 P. FlOURENTZOS, op. cit. (supra, n. 6), p. 12, pl. VI, 15, 16 et pl. XXIV, 55, 56, 58.

22 En Sicile, L. ARTIFA, E. LESNES, " Primi dati sulle produzioni ceramiche palermitane dal $x$ al XV secolo ", in G. DEMIANS D'ARCHIMBAUD (éd.). La céramique médiévale en Méditerranée, Actes du VIe congrès de l'AlECM2, Aix-en-Provence, 13-18 novembre 1995 (1997), p. 411, 412, fig. 3.5. En Égypte, L. MENASA, P. LAFERRIĖRE, La Sàquia. Technique et vocabulaire de la roue à eau égyptienne, Publications de l'IFAO (1975); D. M. BAILEY, "The Pottery from the South Church at El-Ashmunein ", Cahiers de la Céramique Égyptienne 4 (1996), pl. XVII, fig. 34 ; S. MARCHANO, D. LAISNEY, "Le survey de Dendara (1996-1997) ^, Cahiers de la Céramique Égyptienne 6 (2000), nos 208-214. En Tunisie, de nombreux exemples sont conservés dans le dépôt de Raqqada, S. GRAGUEB, Recherches sur deux zones de production de céramiques du haut Moyen Âge en Tunisie: Tunis et Kairouan, thèse en cours, université d'Aix-en-Provence.

23 Des godets fragmentaires de même type, ainsi que des surcuits, avaient déjà été trouvés par Catling. Ils sont publiés par M.-L. VON WARTBURG mais identifiés à tort comme des fragments d'amphores, loc. cit. (supra, n. 2), p. 390, fig. 5.6, 5.7 ; p. 392 , fig. $6.1-4$.

24 M. YON, "Ateliers et traditions céramiques ", in Chypre. La vie quotidienne de l'Antiquité à nos jours, Actes du Colloque, Musée de l'Homme, 1975 (1985), p. 103-114; I. IONAS, Pottery in the Cyprus Tradition (1998). 


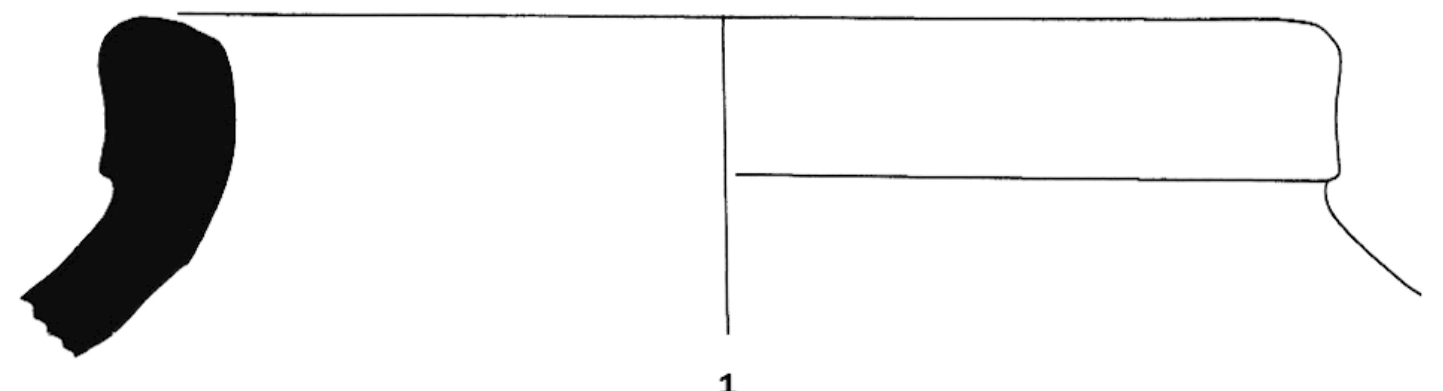

1
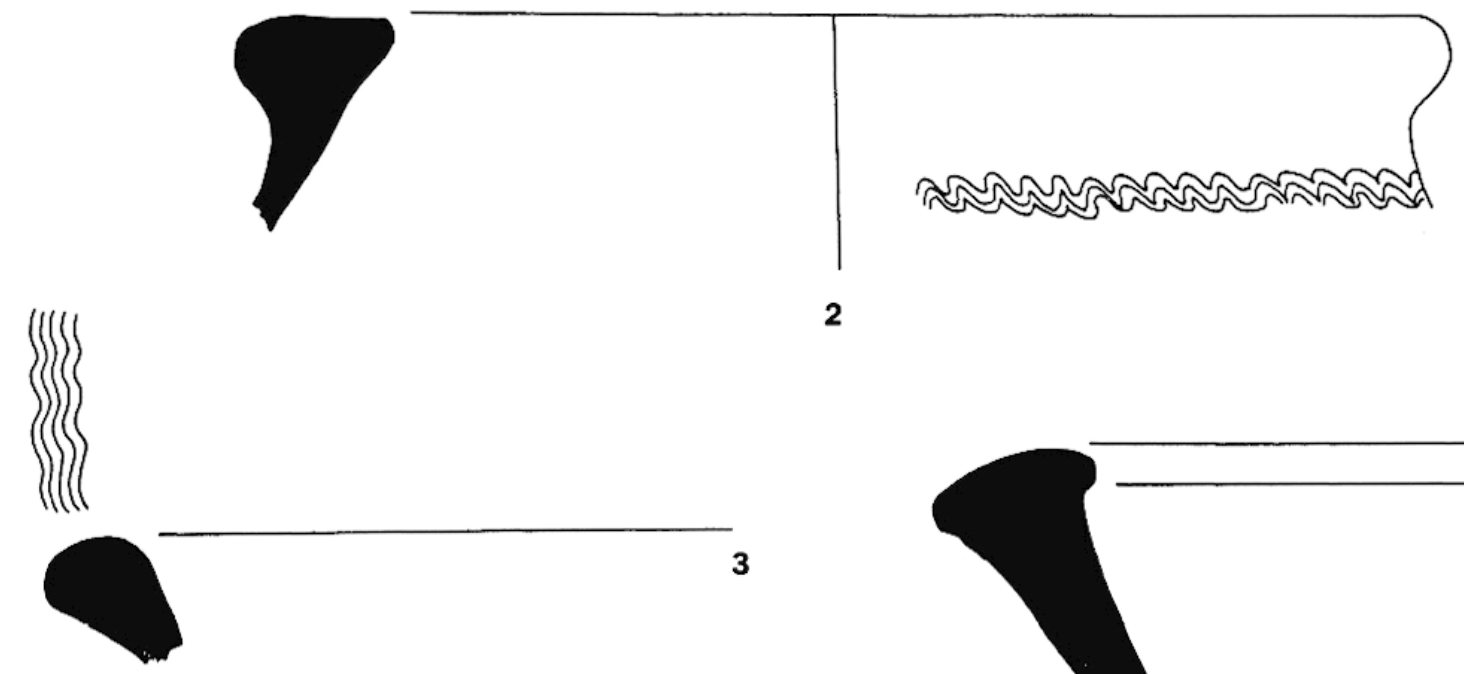

2
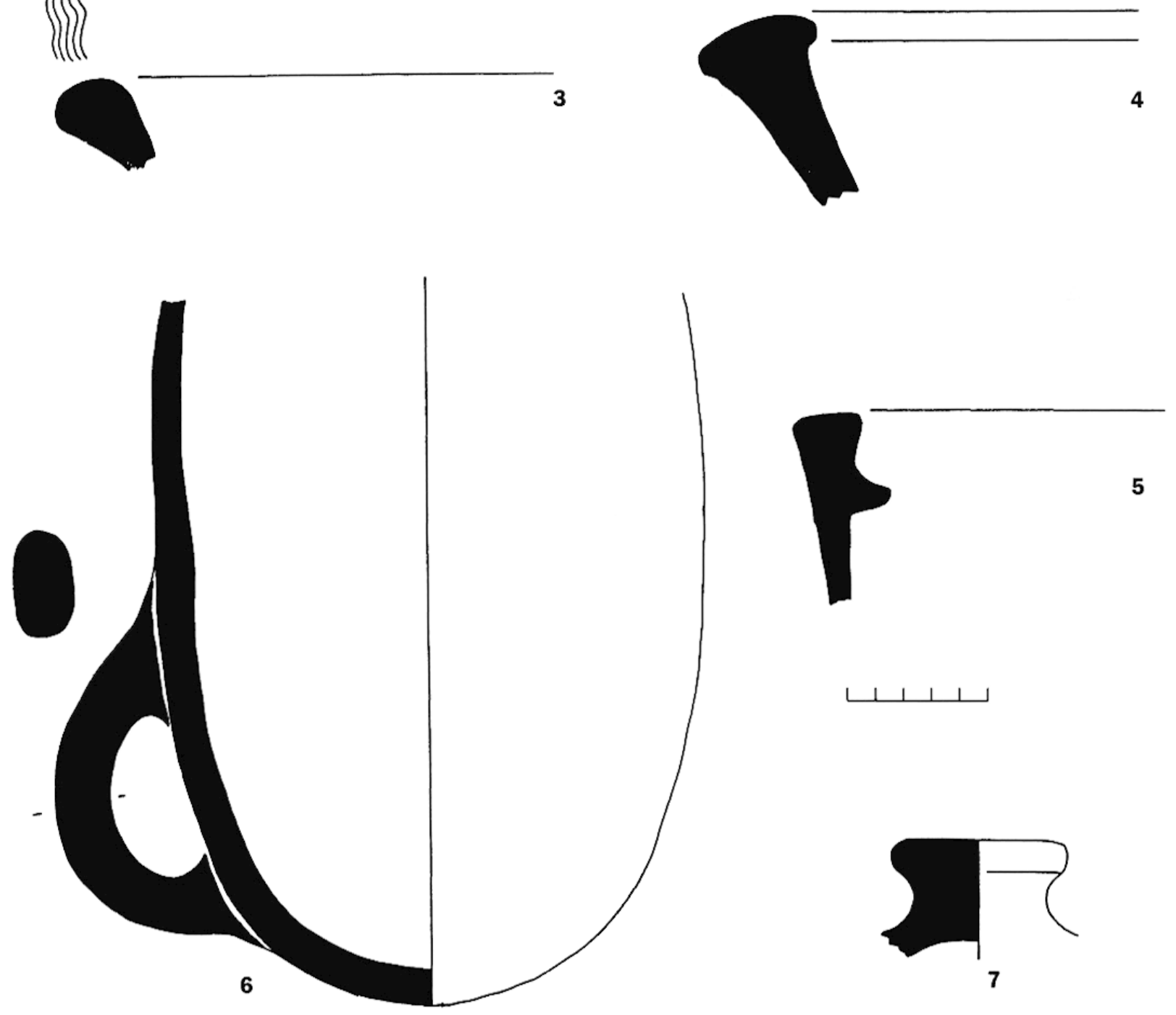

Fig. 3. Céramiques en pâte rouge grossière sans revêtement. 

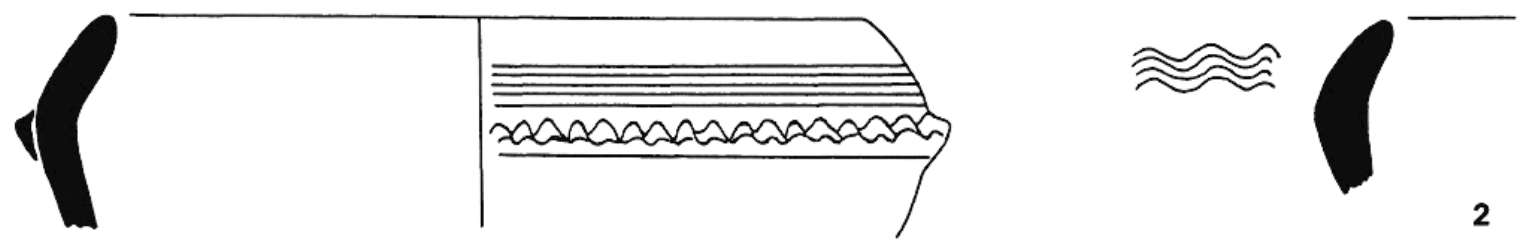

1
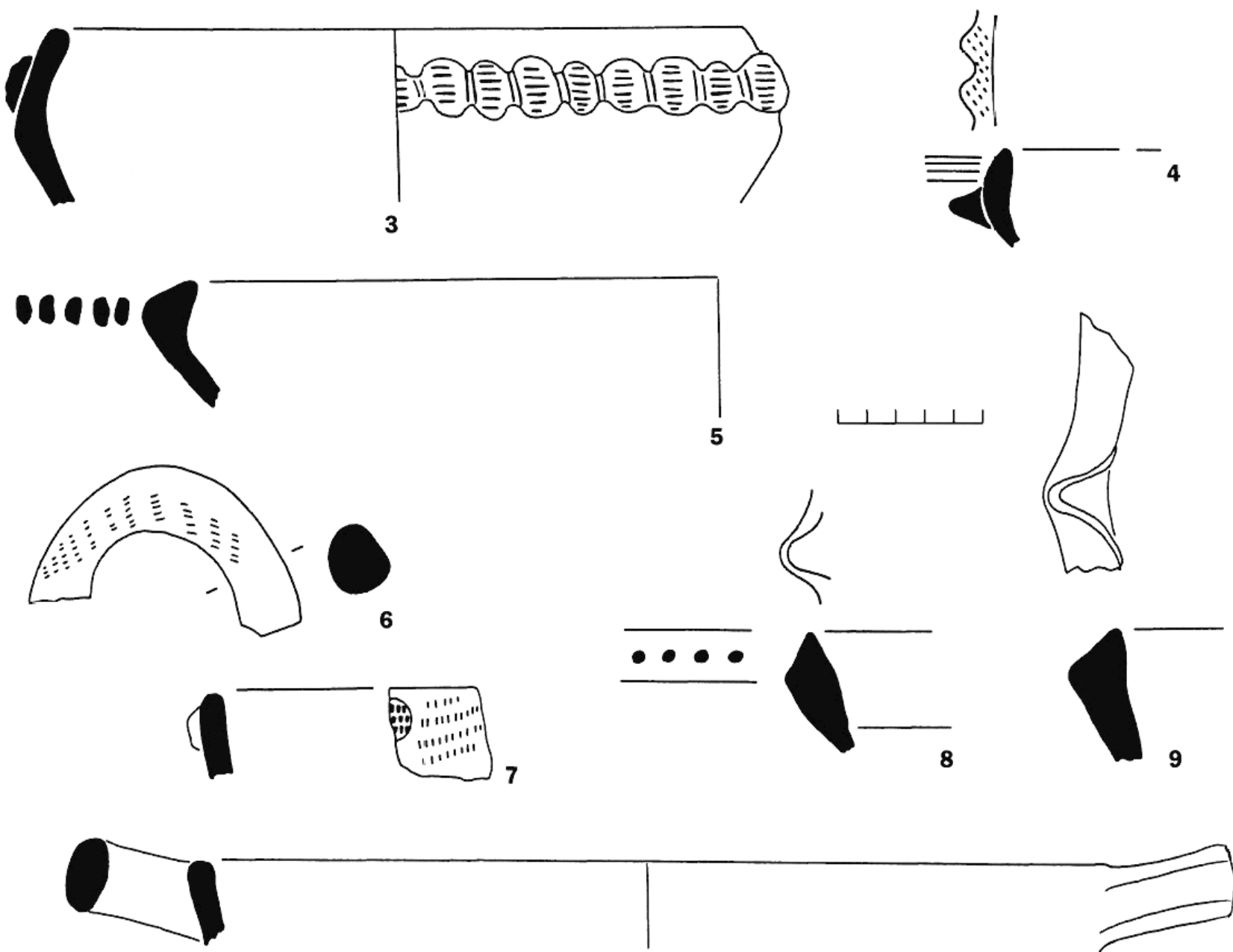

7

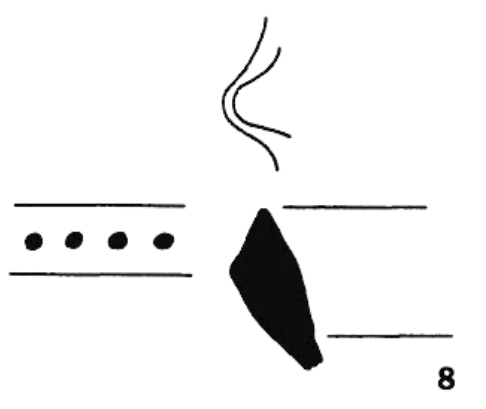

9

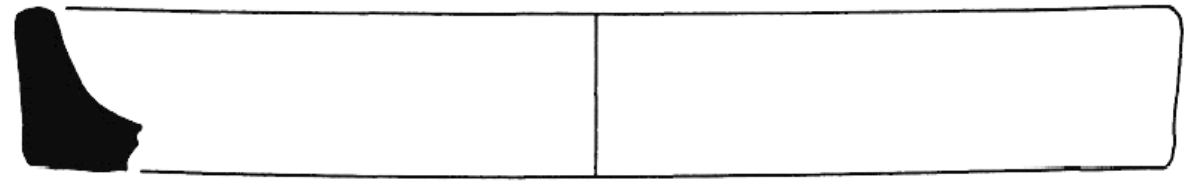

11

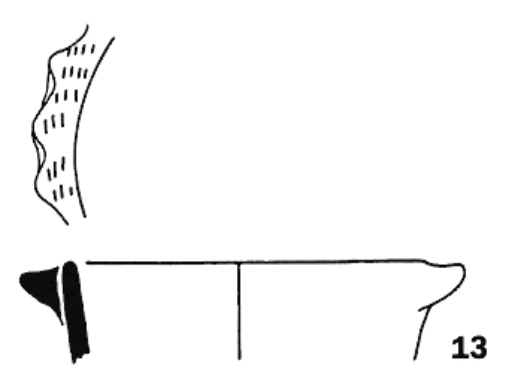

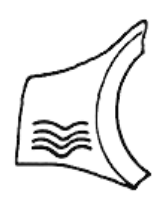

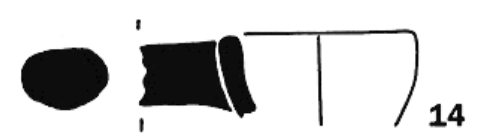

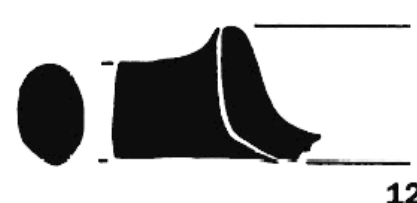
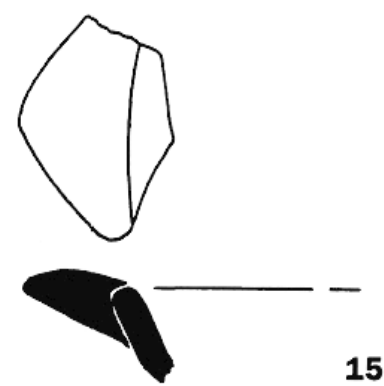

Fig. 4. Céramiques en pâte rouge glaçurées. 
Cependant la permanence des formes retrouvées dans les fouilles et les prospections est confondante; cette poterie est toujours utilisée dans la vie quotidienne, même si elle devient de plus en plus un objet décoratif dans les jardins et les cours. Le stock très imposant vendu dans le village voisin de Mosphiloti conserve le souvenir des productions du siècle dernier et constitue un véritable conservatoire du patrimoine potier de Chypre. Des analyses géochimiques permettraient d'en révéler la composition et de comparer les constituants des pâtes archéologiques à ceux des gisements exploités de nos jours. Le nombre élevé de fragments de bords, de fonds bombés, d'anses et de panses décorées retrouvés dans les parcelles rend bien compte de ces divers usages. Des bords épais en bourrelet ou aplatis pourraient appartenir à des cols de jarres et de vases de stockage sphériques (fig. 3, $\mathrm{n}^{\text {os }}$ 1-4). Cette forme de pithos a été retrouvée en place dans la cour d'une maison à Kouklia datée par une monnaie de 1571. D'après F. G. Maier, elle appartient à la catégorie des céramiques modelées en pâte rouge à décor incisé, retrouvée en grand nombre avec les sgraffito tardifs de Lapithos ${ }^{25}$. La même argile a aussi servi à fabriquer des couvercles à gros bouton de préhension ainsi que des pots de noria d'un autre type que celui en argile calcaire avec des fonds ronds et deux anses superposées pour leur fixation à la roue (fig. $3, \mathrm{n}^{\text {os }} \mathbf{6 , 7}$ ). Des exemplaires bien conservés ont pu être identifiés parmi les tessons mis au jour dans les fouilles pratiquées par $\mathrm{D}$. Michaelidès dans la chapelle de l'Archange Michel ${ }^{26}$. Ces grosses formes pour la conservation des liquides n'ont pas de revêtement. La céramique culinaire est plus abondante et variée dans les formes. La glaçure est présente sur la plupart des vases allant au feu. Certains objets, comme les poêlons bas, sont munis d'anses horizontales, tandis que les jattes tronconiques à bec verseur possèdent des anses verticales (fig. 4, $\mathrm{n}^{\text {os }}$ 1-12). Ces deux types d'ustensiles sont totalement couverts de glaçure à l'intérieur et les exemplaires complets issus du puits de Nicosie et de Palaipaphos confirment l'usage de ces céramiques communes dès le XIII et le XIVe siècle. Une lèvre épaisse à ressaut interne pourrait appartenir à un brasero (fig. $3, n^{\circ} 5$ ). Les pots, de dimensions variables, pour les liquides ou la cuisson, sont munis d'anses verticales en boudin appliquées directement sur la lèvre du col et parfois aussi d'un bec tubulaire (fig. 5, nos 1-10). Ils sont partiellement glaçurés à l'embouchure et à l'intérieur. Des petites formes, peut-être des coupelles de lampes à huile ou d'encensoirs, possèdent des languettes de préhension (fig. 4, $\mathrm{n}^{\text {os }}$ 13-15). Cette production en argile rouge lie-de-vin, particulièrement typique, est ornée avec beaucoup de soin. On trouve des cordons digités ou découpés, des pastilles appliquées, des répétitions d'impressions réalisées à la roulette, au peigne, ou avec des tiges creuses juxtaposées dans des jeux de compositions complexes d'ondes, de lignes, de rouelles, d'oculi, de hachures (fig. 4 et 5 ; fig. 9, no 3 ). Si la céramique en pâte rouge semble bien illustrée dans toute l'île, notamment à Paphos, la dualité de production de céramiques communes, en pâte claire et en pâte rouge, bien différentes mais complémentaires, se retrouve plus spécifiquement dans les fouilles de Nicosie ${ }^{27}$.

25 F. MAIER, * Excavations at Kouklia (Palaipaphos), Tenth Preliminary Report: Seasons 1977 and 1978 ", RDAC 1979, p. 172, pl. XVI, 4, XVII, 2.

26 Cf. N. LECUYER et al., infra, p. 672-674.
27 D. PAPANIKOLA-BAKIRTZIS (1999), op. cit. (supra, n. 17), fig. 29-31; P. FLOURENTZOS, op. cit. (supra, n. 6), pl. VII, XXII, $n^{\text {os }} 46,51$ et pl. XXIII; F. GIUDICE et al. (1996), loc. cit. (supra, n. 14), p. 252, cat. C56. 

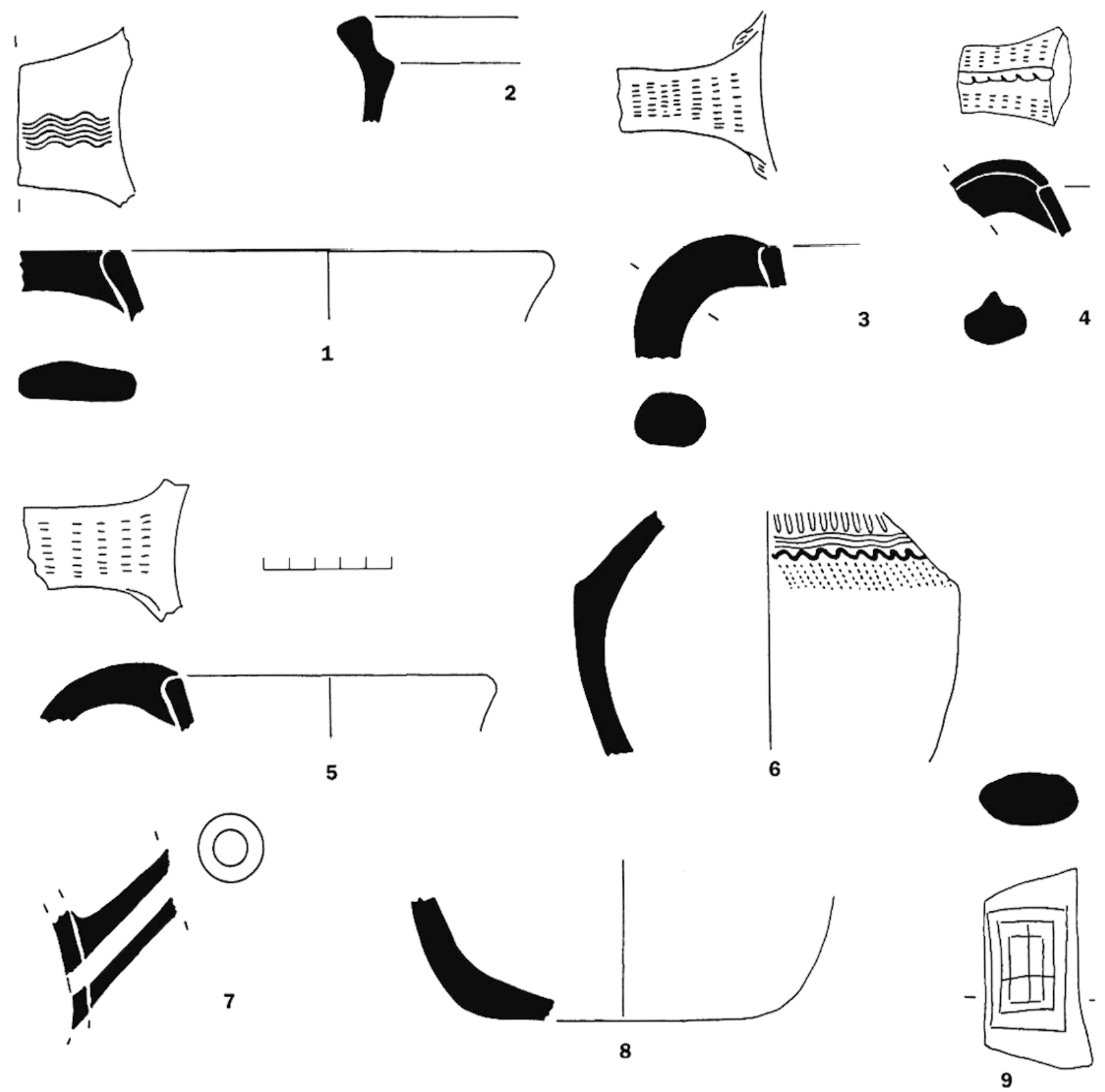

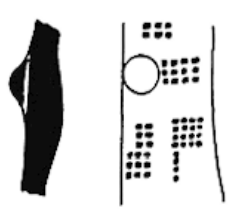

10

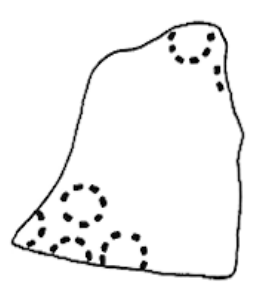

15

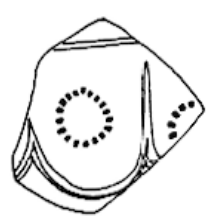

11

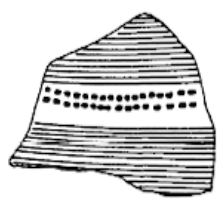

12

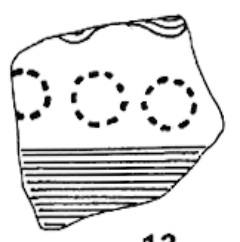

13

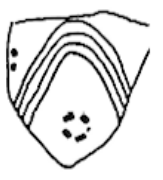

14
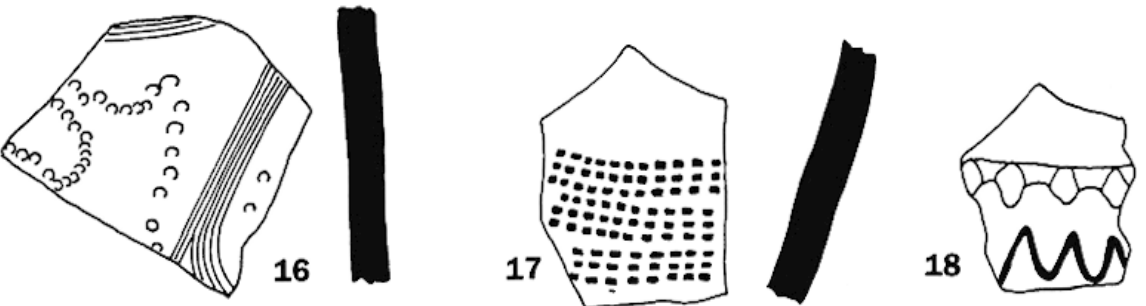

Fig. 5. Céramiques en pâte rouge glaçurées. 

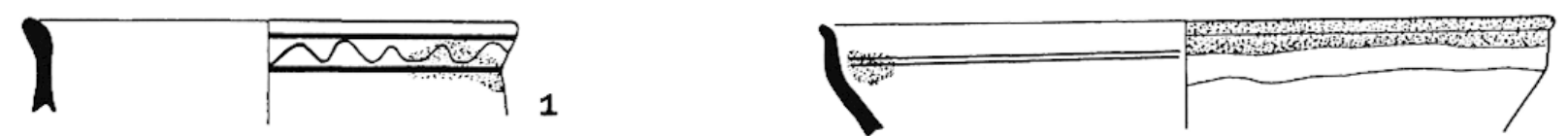

4
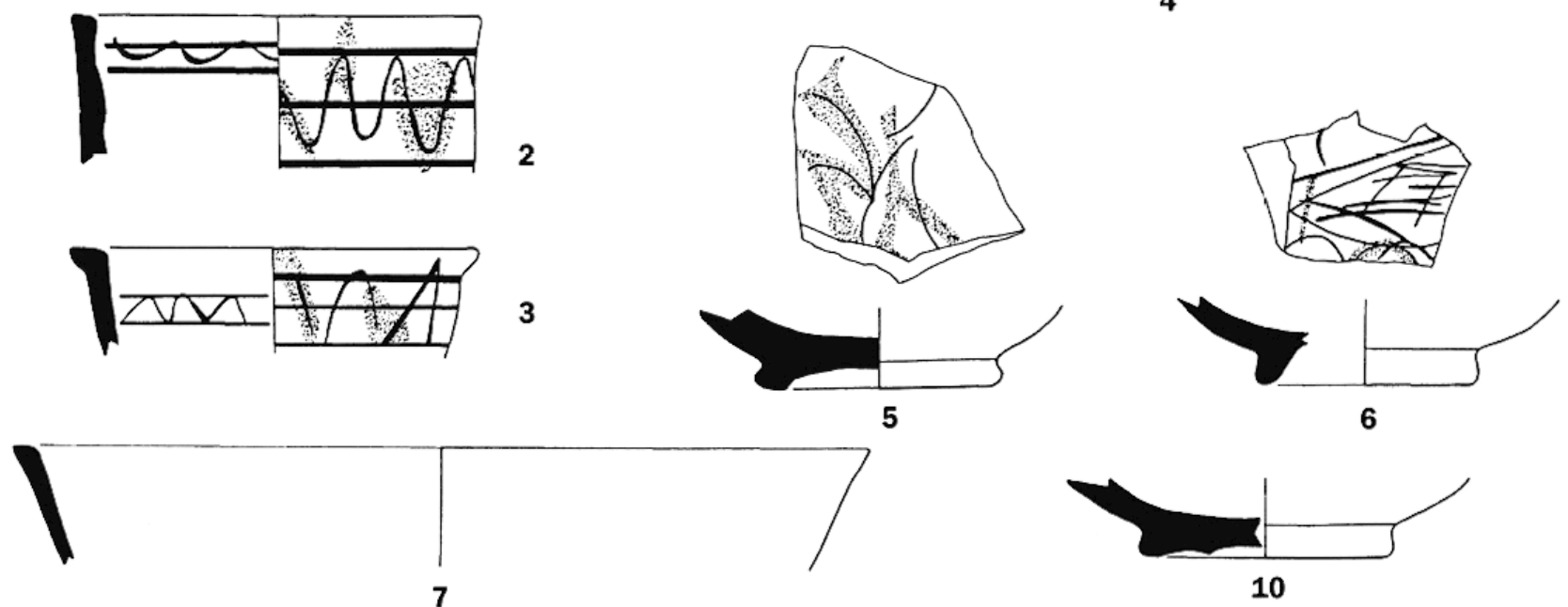

7

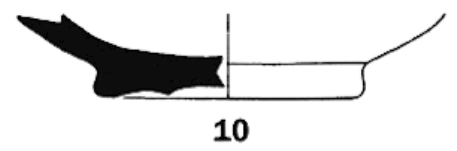

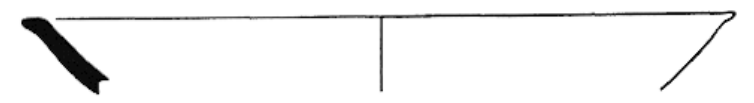

8

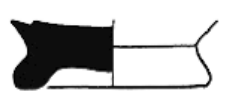

11

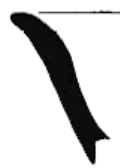

9
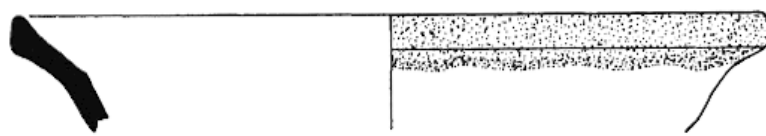

16
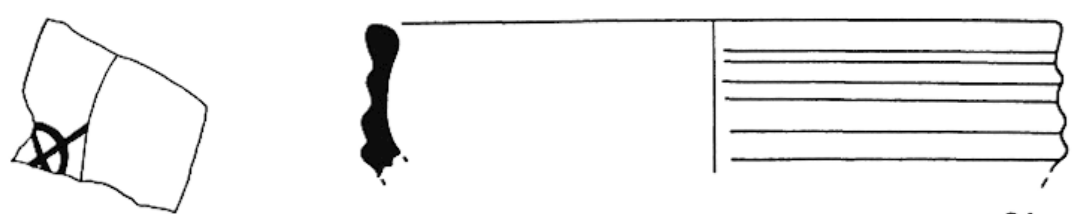

21
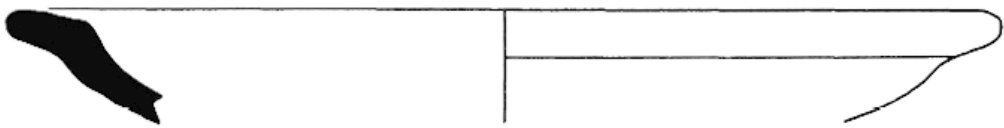

15

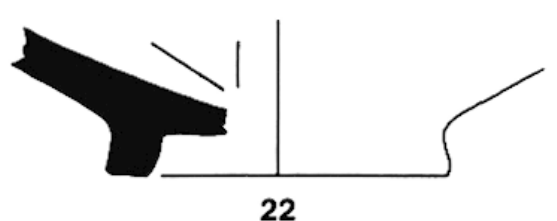

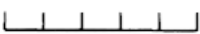

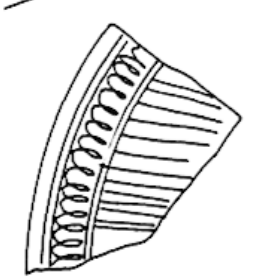

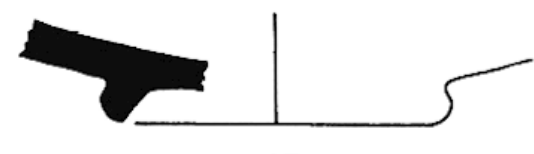

18

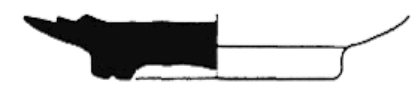

12
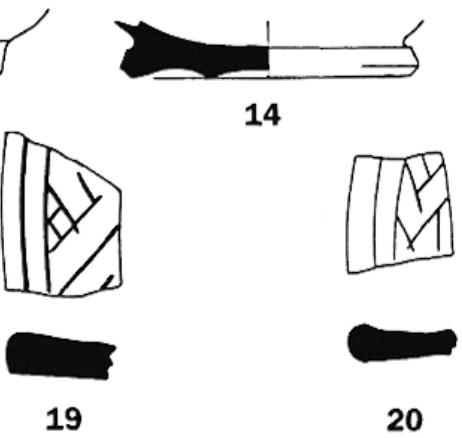

17

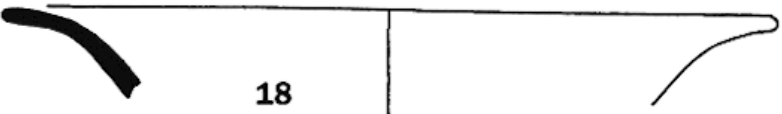

Fig. 6. Vaisselle de table de Lapithos (1-14); Aegean Ware byzantine (15); graffita d'Italie du Nord (17-22).

BCH 125 (2001) 


\section{A. 2. Vaisselle de table chypriote}

A Potamia, la vaisselle de table médiévale - c'est-à-dire des coupes, des plats et des ciboires à pâte rouge, engobés et glaçurés — représente $37 \%$ des découvertes pour la période. Cette céramique provient en grande partie de deux ateliers chypriotes dont les productions sont bien connues. Les vases issus des officines de la région de Paphos, qui fonctionnent du XIII siècle au début du XIVe, sont très rares sur le site, soit $1 \%$ du matériel. Il s'agit de fragments de coupes au décor finement incisé - des fleurons polylobés se détachant sur fond de boucles - sous rehauts polychromes verts et brun ${ }^{28}$ (fig. $10, \mathrm{n}^{\circ} 1$ ). Mais l'essentiel de la vaisselle fine utilisée (19\%) est issue des officines de Lapithos, en activité de la fin du XIVe siècle au début du XVI ${ }^{e}$. Trois phases de production sont représentées parmi les trouvailles de la prospection. Un premier groupe rassemble des céramiques incisées peintes polychromes ${ }^{29}$ datées de la fin XIVe-XVe siècle (12\%) (fig. 10, $n^{\circ} 2$ ). Ce sont notamment des fragments de calices, dont la paroi extérieure est ornée de cercles concentriques et de lignes ondulées (fig. 6, $\mathbf{n}^{\text {os }} 1$ 3 ), et des coupes décorées de branchages stylisés; ces décors, rendus par une économie de traits, sont tracés librement et rapidement à l'intérieur comme à l'extérieur. Les coupes incisées peintes monochromes (fig. 6, nos 4-6), attribuées à la fin XVe-début du XVI siècle, sont moins nombreuses $^{30}(2 \%)$. La composition qui prédomine consiste en un médaillon orné d'éléments végétaux ou géométriques incisés finement, enfermé dans un carré aux côtés concaves repris par des bandes peintes en vert (fig. $10, n^{\circ} 3$ ) ; la glaçure est incolore. La dernière phase de production médiévale de Lapithos représente sur le site $5 \%$ du matériel : il s'agit de coupelles à parois fines, à lèvres légèrement éversées, tournées dans une pâte fine, rose (fig. 6, $\mathrm{n}^{\text {os }} 7-9$ ). L'anneau des bases annulaires est large et très plat (fig. 6, $\mathrm{n}^{\text {os }}$ 10-14). Les décors géométriques - des lignes ondulées, des points et des zigzags - sont peints en vert ou brun sur un engobe très délayé couvert par une glaçure brillante très pauvre ${ }^{31}\left(\mathrm{fig} .11, \mathrm{n}^{\circ} 1\right)$. Cette poterie est considérée comme une forme décadente de la série précédente. Enfin, un nombre assez important de vases chypriotes à glaçure monochrome $(17 \%)$ complète les découvertes. Leur état très fragmentaire empêche toute attribution aux ateliers connus, ce qui plaide en faveur d'une fabrication sinon locale, du moins régionale.

28 Type Brown and Green Incised Sgraffito Ware de D. PAPANIKOLA-BAKIRTZIS (1996), op. cit. (supra, n. 17). 29 Type Brown and Green Sgraffito Ware, ibid.
30 Type Green Painted Sgraffito Ware, ibid. 31 Type Green Painted Ware, ibid. 


\section{A. 3. Productions de Méditerranée orientale et occidentale}

La vaisselle fine médiévale d'origine étrangère ne représente, à Potamia, que $1 \%$ du matériel glaçuré. La grande diversité des types identifiés contraste avec le faible volume de ces importations.

Un fragment unique de céramique byzantine, de type Aegean Ware ${ }^{32}$, illustre la production la plus ancienne, c'est-à-dire le début du XIII siècle. C'est une coupe à petit marli, largement ouverte, à pâte orange assez fine, avec pour décor un cercle barré d'une croix réalisé dans une incision large et couvert d'une glaçure jaunâtre (fig. $6, \mathrm{n}^{\circ} 15$ ). Ce type d'objet, dont l'origine est inconnue, est très largement diffusé en Méditerranée, et à Chypre on en trouve la trace dans les fouilles de Saranda Kolones à Paphos et dans celles du sanctuaire d'Aphrodite à Palaipaphos $^{33}$. La quasi-absence de vaisselle byzantine à Potamia n'a rien de surprenant, la céramique produite dans l'empire étant rarement attestée dans les fouilles chypriotes dans lesquelles, en plus de l'Aegean Ware, on trouve occasionnellement du sgraffito fin et de la Zeuxippus Ware mais toujours en quantité faible. Si cette dernière catégorie n'est pas représentée parmi notre matériel, nous avons en revanche isolé des imitations chypriotes de ce type, quelques bords de coupes à pâte orangée, fine et dure, ornées de cercles concentriques incisés sous une glaçure jaune pâle très brillante (fig. 6, $\mathrm{n}^{\circ}$ 16). Des analyses physico-chimiques de pâte menées sur des objets de même aspect ont montré que ces vases étaient fabriqués dans les ateliers de Paphos ${ }^{34}$.

L'essentiel des importations provient du bassin occidental de la Méditerranée. Un premier groupe est constitué de quelques fragments de céramique espagnole émaillée à décor peint au bleu de cobalt, issus des ateliers de Valence - des faïences des XIV ${ }^{e}$-XVe siècles dont l'aire de distribution est très vaste ${ }^{35}$. Un deuxième groupe rassemble diverses productions originaires d'Italie. Parmi elles, on trouve des céramiques haut de gamme incisées sous glaçure plombifere, fabriquées en Italie du Nord aux XVe et XVIe siècles, comme ce tesson avec une représentation d'un homme enturbanné figuré de profil, un décor incisé rehaussé de pigments brunâtres et bleus (fig. $6, \mathrm{n}^{\mathrm{os}} 17,18$ ). Style et coloris laissent croire à une origine de Lombardie ou de Vénétie ${ }^{36}$. Des coupelles de même type ont été retrouvées sur d'autres sites chypriotes: à Nicosie, des coupes ornées de rinceaux végétaux excisés, d'animaux et de portraits incisés, sont attribuées aux ateliers de Bologne ${ }^{37}$ pour le

32 Sur ce type, voir notamment, G. THEOPHILOU, M. MICHAIILL-

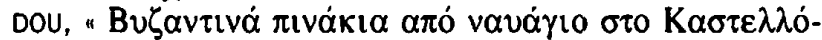
plלo ", $A D 41$ (1986) [1991], A', p. 271-328, fig. $79 \alpha$; et sur sa distribution, V. FRANÇOIS, "Sur la circulation des céra miques byzantines en Méditerranée orientale et occidentale ", in G. DEMIANS D'ARCHIMBAUD (éd.), op. cit. (supra, n. 22), p. 231-236.

33 A. H. S. MEGAW (1971), loc. cit. (supra, n. 12), p. 125, pl. XXXIII, 4 ; id., "Supplementary Excavations on a Castle Site at Paphos, Cyprus, $1970-71$ n, DOP 26 (1972), p. 322 343 , nos $28,31,33 ;$ M.-L. VON WARTBURG, loc. cit. (supra, n. 13), p. 186, fig. 10, pl. XX, 7,8 .
34 Résultats préliminaires d'un programme de recherche en cours sur la Zeuxippus Ware et ses dérivés mené par Y. Waksman (CNRS-UPR 7524) et V. François.

35 Pour une recension des découvertes par aire géographique, voir H. BLAKE, * La ceramica medievale spagnola e la Liguria ", Atti del V Convegno Internazionale della Ceramica (1972), p. 80-83; V. FRançoIs, Céramiques médiévales à Alexandrie, Études Alexandrines 2 (1999), p. 171-172.

36 S. NEPOTI, Ceramiche graffite della donazione Donini Baer (1991), tav. XXXIV.

37 J. DU PLAT TAYLOR, A. H. S. MEgaw, loc. cit. (supra, n. 11), p. 12 , pl. IX, 2, 4, n' 27. 


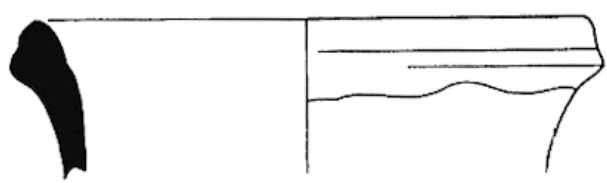
1
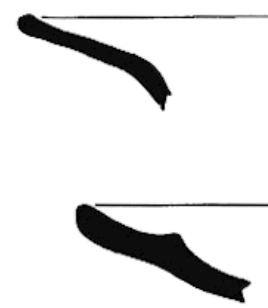

$\checkmark$

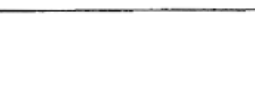

4

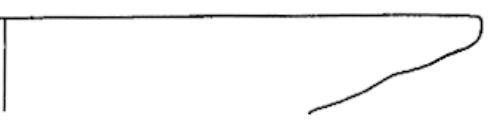

5

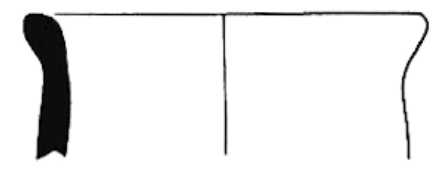

2

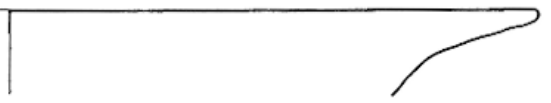

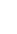

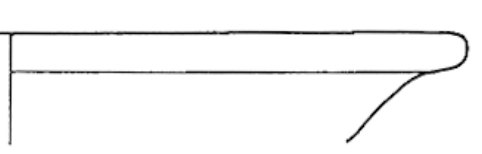

6
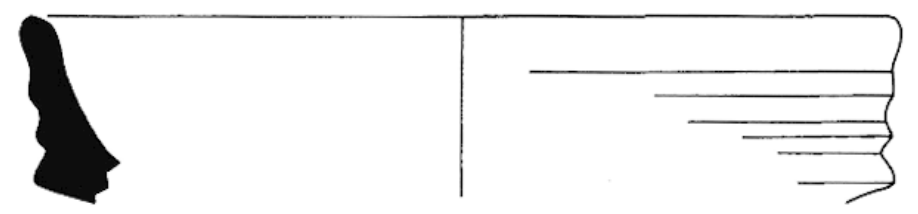

9
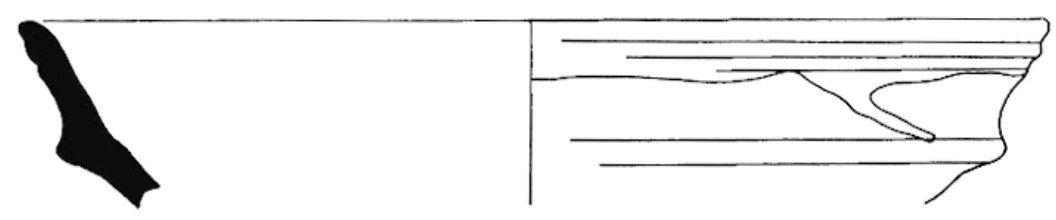

10
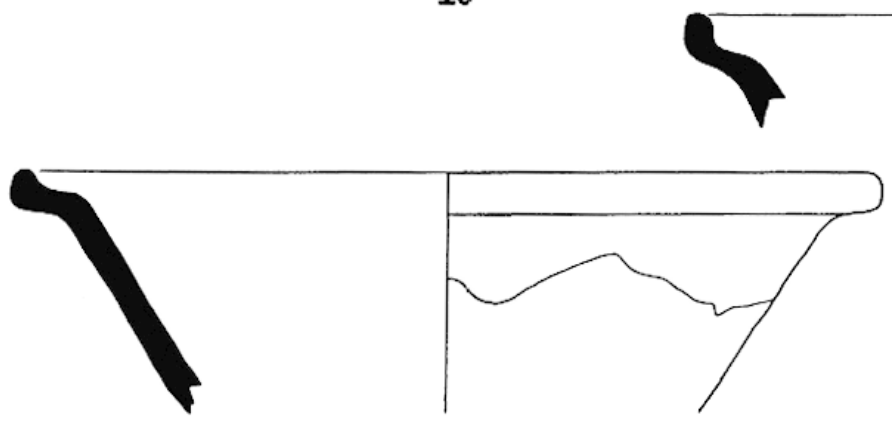

12
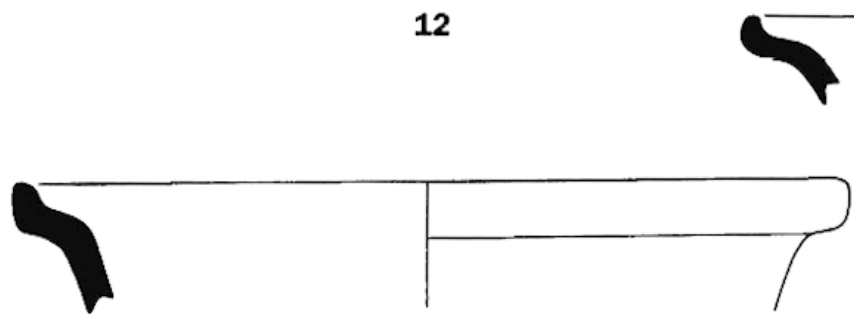

14

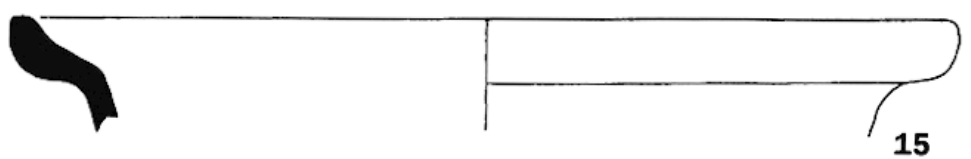

Fig. 7. Céramiques chypriotes glaçurées d'époque ottomane. 
$\mathrm{XV}^{\mathrm{e}}$ siècle; à Famagouste, un fragment avec un soldat casqué est identifié comme un exemplaire de la production padane ${ }^{38}$ du XVI siècle; un angelot représenté sur fond de décor architectural antique, typique de la Renaissance, orne une coupe découverte à Palaipaphos, datée entre le XVe et le début du XVI siècle et provenant vraisemblablement des officines de Padoue ou d'Aquilée ${ }^{39}$; enfin, dans le musée du manoir royal des Lusignan, à Kouklia, une coupelle décorée d'un lièvre incisé sous rehauts polychromes, datée de la deuxième moitié $\mathrm{du} \mathrm{XV}^{\mathrm{e}}$ siècle, viendrait d'Émilie ${ }^{40}$. D'autres productions d'Italie septentrionale apparaissent également parmi les importations recueillies lors de la prospection: un fragment de panse d'une grande coupe ornée à l'extérieur de trois ressauts imprimés à la roulette ${ }^{41}$ (fig. $6, \mathbf{n}^{\circ} 21$ ) semblable à un vase complet de Paphos ${ }^{42}$ ainsi qu'aux coupes du sanctuaire d'Aphrodite de Palaipaphos ${ }^{43}$; des coupes dites a graffita monochroma, en pâte orange recouverte d'engobe et de glaçure jaune ou orangée, qui proviennent de Ligurie, probablement des officines de Savone ${ }^{44}$ (fig. 6, $\mathrm{n}^{\text {os }}$ 19-22). C'est également aux ateliers ligures qu'on peut attribuer quelques fragments d'assiettes, avec ou sans marli, en faïence, ornées d'un décor floral très fin d'inspiration orientale dit calligrafico a volute tipo $C$, peint en bleu foncé sur un fond blanc ou bleu pâle ${ }^{45}$. Cette production datée du XVI ${ }^{\mathrm{e}}$ siècle apparaît également à Nicosie ${ }^{46}$ et parmi les collections du musée de Limassol. D'autres faïences, mais cette fois des cruches pansues ornées d'un décor peint polychrome, venues des officines toscanes de Montelupo, complètent l'inventaire des importations italiennes pour la période. Un très bel exemplaire de ce type, presque complet, provient d'Agia Photou à Nicosie ${ }^{47}$, tandis que de mêmes vases à liquides, fragmentaires cette fois, ont été mis au jour dans les fouilles de Garrison's Camp à Paphos ${ }^{48}$. Ces productions italiennes des XVe$\mathrm{XVI}^{\mathrm{e}}$ siècles sont des types bien attestés sur d'autres sites du Proche-Orient. Aussi ne serait-il pas raisonnable de justifier leur présence par l'occupation vénitienne de l'île. Ce sont les marines marchandes italiennes, fort présentes en Méditerranée orientale, qui ont largement contribué à la diffusion de tels produits.

\section{B. Période ottomane}

Les découvertes de céramiques communes à glaçure et de vaisselle de table, datées entre la fin du XVIe siècle et le début du XIXe, représentent seulement $3 \%$ du total des fragments recueillis.

38 T. MOGABGAB, "Excavations and Researches in Famagusta, 1937-1939 ^, RDAC 1937-1939 [1951], p. 190, pl. XXXVI, no 4. 39 M.-L. VON WARTBURG (1997), loc. cit. (supra, n. 13), p. 194, pl. XXI, nos 6,7 .

40 Pour des exemples proches, voir S. NEPOTI, op. cit., (supra, n. 36), p. 191, n 17, p. 193, n²3, p. 195, nº 27.

41 G. B. SIVIERO, "La ceramica graffita venete ", Atti del XIX Convegno Internazionale della Ceramica (1986), p. 32.

42 F. GIUDICE et al. (1993), loc. cit. (supra, n. 14), p. 290 , $n^{\circ} 29$, fig. 13, no 1 , tav. LXXXV, no 2.
43 M.-L. VON WARTBURG (1997), loc. cit. (supra, n. 13), p. 194, fig. $19, n^{\circ} 1,2$, pl. XXI, no 6 .

44 H. AMOURIC, FI. RICHEZ, L. VALLAURI, Vingt mille pots sous les mers (1999), p. 64-66.

45 Ibid., p. 63, fig. 137-139.

46 J. DU PLAT TAYLOR, A. H. S. MEGaW, loc. cit. (supra, n. 11), pl. IX, 4, nos 24, 29.

47 T. MOGABGAB, loc. cit. (supra, n. 38), p. 190, pl. XXXVI, no 7. 48 F. GIUDICE et al. (1993), loc. cit. (supra, n. 14), p. 295, fig. $15, n^{\circ} 7$, tav. XC. 

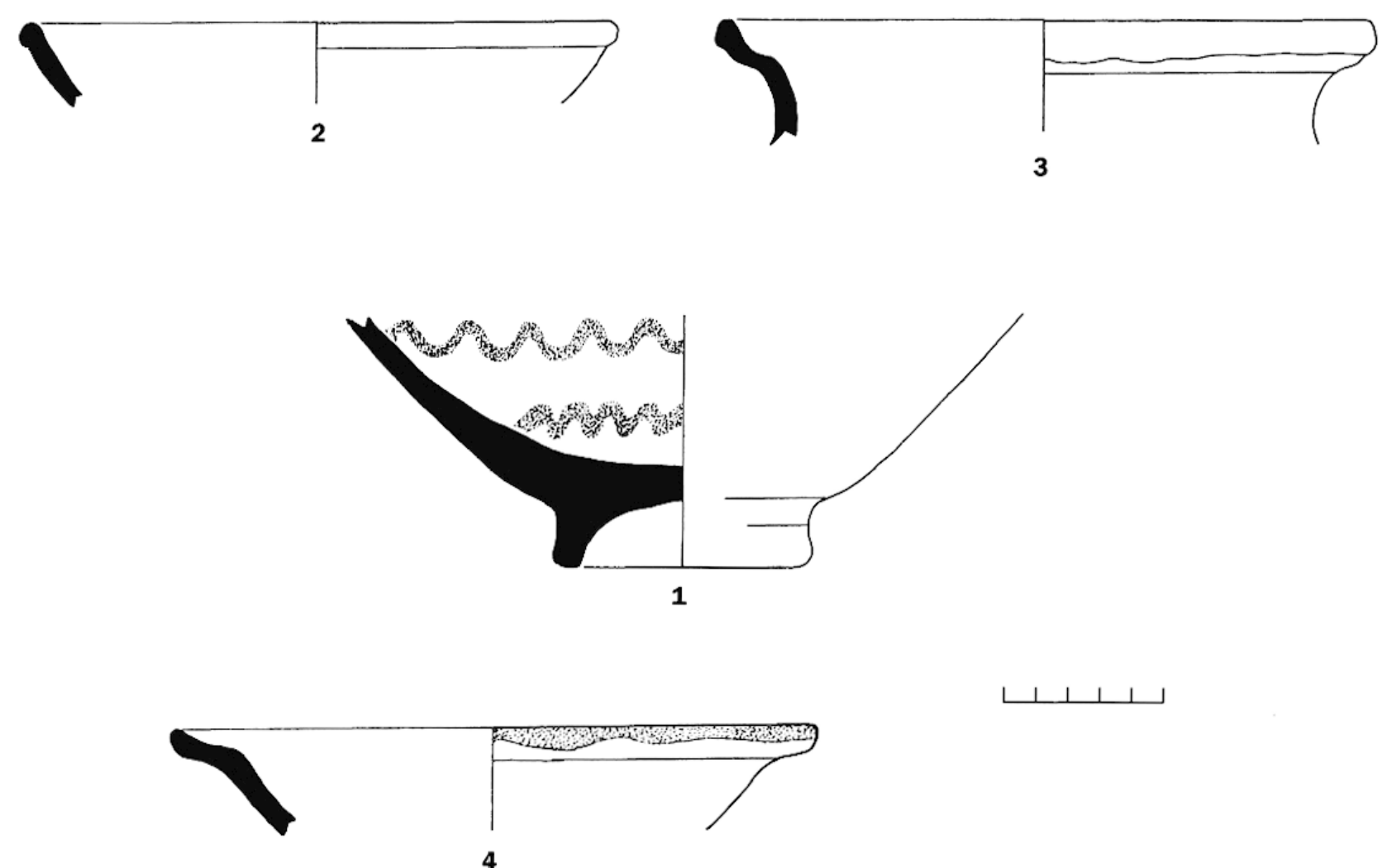

4

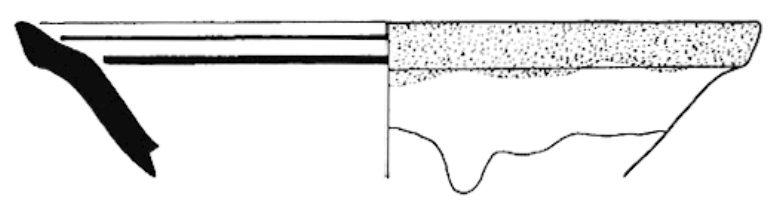

5

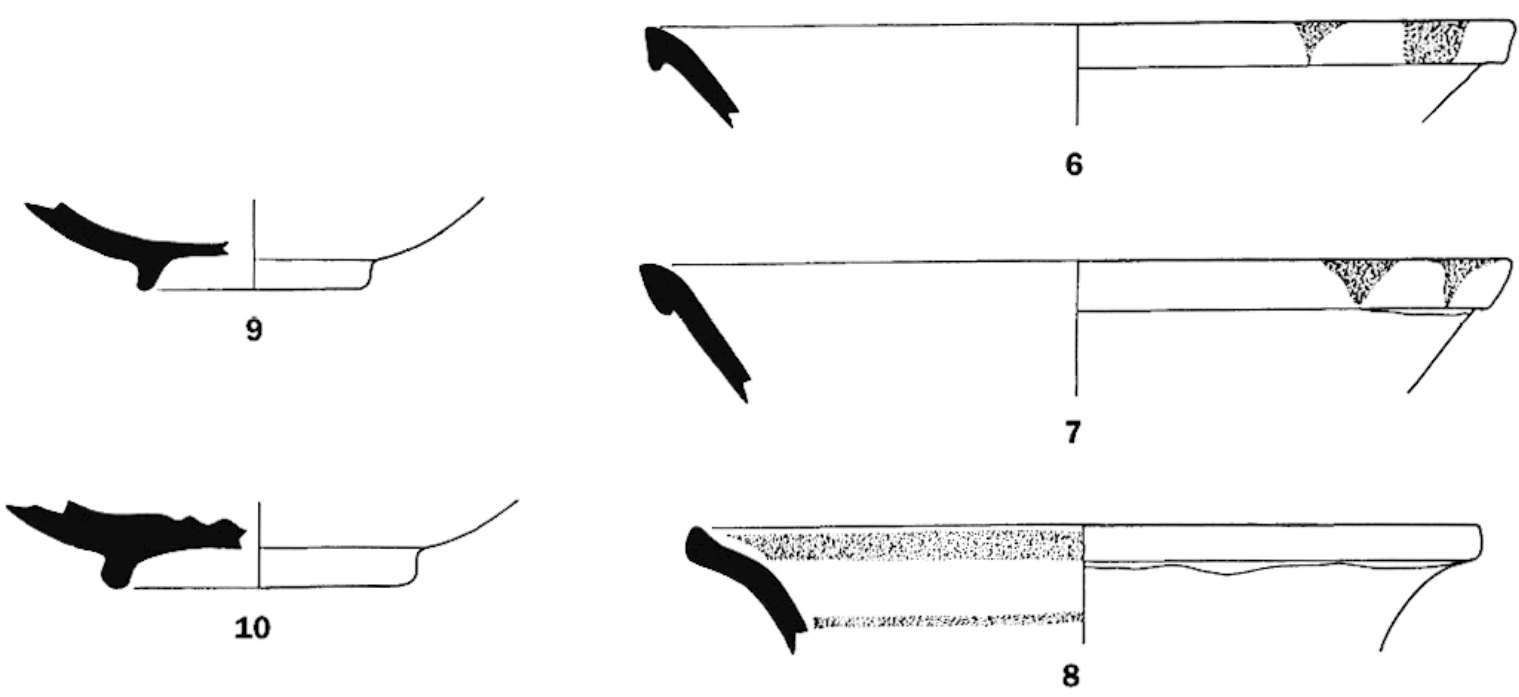

Fig. 8. Céramiques chypriotes glaçurées d'époque ottomane (1-3); céramiques de Çanakkale $(4,5)$; céramiques de Didymotique (6-10). 


\section{B.1. Vaisselle commune glaçurée}

Il a été montré, à partir du matériel mis au jour lors des fouilles de Saraçhane à Istanbul, qu'à l'époque ottomane les céramiques à glaçure plombifere se substituent à la vaisselle culinaire à paroi fine ${ }^{49}$, une constatation confirmée par les découvertes d'Aphrodisias ${ }^{50}$ et par celles de Potamia, où l'on recense toute une série de vases glaçurés d'aspect assez grossier. Parmi eux, retenons des cols de jarre et de cruche à bec tubulaire - ibrik - un fragment de pot de chambre ou de pot pour chaise percée avec un large bord éversé, la base plate d'un pot, celle convexe d'une jatte, et un couvercle qui s'incurve en un profil hémisphérique pour former un bouton central plein (fig. $7, \mathbf{n}^{\text {os }} 1-4,7,8$ ). On trouve aussi des coupelles assez plates avec un marli quasiment horizontal, souligné par une arête vive, et de larges çoupes avec à l'extérieur des ressauts imprimés à la roulette (fig. $7, \mathrm{n}^{\circ 5} 5,6,9,10$ ). Ce type déjà isolé par M.-L. von Wartburg à Kouklia pourrait être, selon son hypothèse, une imitation chypriote d'importations d'Italie septentrionale ${ }^{51}$. Une des différences majeures permettant de distinguer les productions médiévales des productions ottomanes est la taille des vases, qui augmente considérablement. C'est pour cette raison que la grande coupe avec un décor de lignes ondulées peint à l'engobe peut être assignée à cette période tardive (fig. $8, \mathbf{n}^{\circ} 1$ ). Il est regrettable que la vaisselle utilisée à Chypre à l'époque de la domination de la Porte ait suscité jusqu'à présent si peu d'intérêt. Bien qu'on ne connaisse pas d'atelier en activité sur l'île du XVII ${ }^{e}$ au XIX siècle, excepté peut-être Lapithos, il nous paraît cependant vraisemblable d'envisager pour tous ces objets une origine chypriote. $\mathrm{Ce}$ qui peut être également le cas pour une autre série, essentiellement constituée de coupelles profondes à petits marlis à lèvre redressée, tournées dans une pâte fine et très dure de couleur orange ou beige (fig. $7, \mathrm{n}^{\text {os }} 11-15 ;$ fig. $8, \mathrm{n}^{\text {os }} 2,3$ ). Une glaçure assez pauvre, verdâtre à jaunâtre, est tantôt appliquée sur une mince couche d'engobe, tantôt directement sur la pâte. Il est possible qu'elles soient les représentantes d'une production tardive de Lapithos, comme le laissent supposer les fabrications du siècle dernier.

\section{B.2. Vaisselle fine et céramique de cuisine et de stockage importées}

Alors que pour l'époque médiévale les vases étrangers sont rares, pour la période ottomane, les importations sont un peu plus nombreuses puisqu'elles s'élèvent à $34 \%$ du matériel. Elles proviennent à la fois d'ateliers de l'empire - de Turquie et de Grèce - et d'officines d'Italie et du Sud de la France.

49 J. W. HAYES, Excavations at Sarachane in Istanbul, II. The Pottery (1992), p. 233.
50 V. FRANÇOIS, "Éléments pour l'histoire ottomane d'Aphrodisias: la vaisselle de terre ", Anatolia Antiqua 9 (2001), p. $147-190$.

51 M.-L. VON WARTBURG (1997), loc. cit. (supra, n. 13), p. 194. 
bd $2001.125 .210=\sin 2$
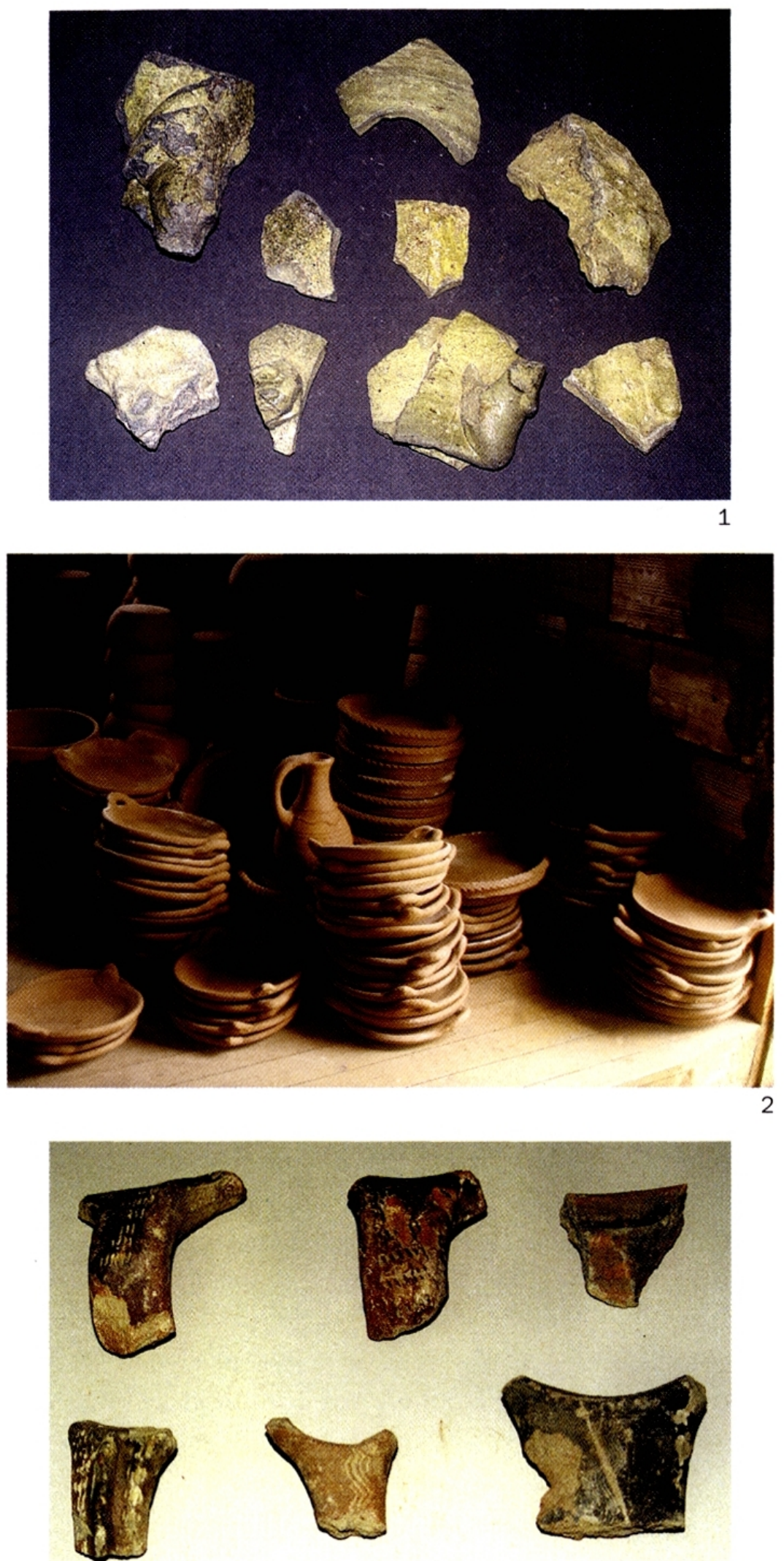

3

Fig. 9. Formes surcuites et déformées de céramiques à pâte calcaire (1); production actuelle de Kornos (2); anses à pâte rouge glaçurées (3). 


$$
\text { bon } 200^{\prime}-80.2 ! \circ ; 53
$$

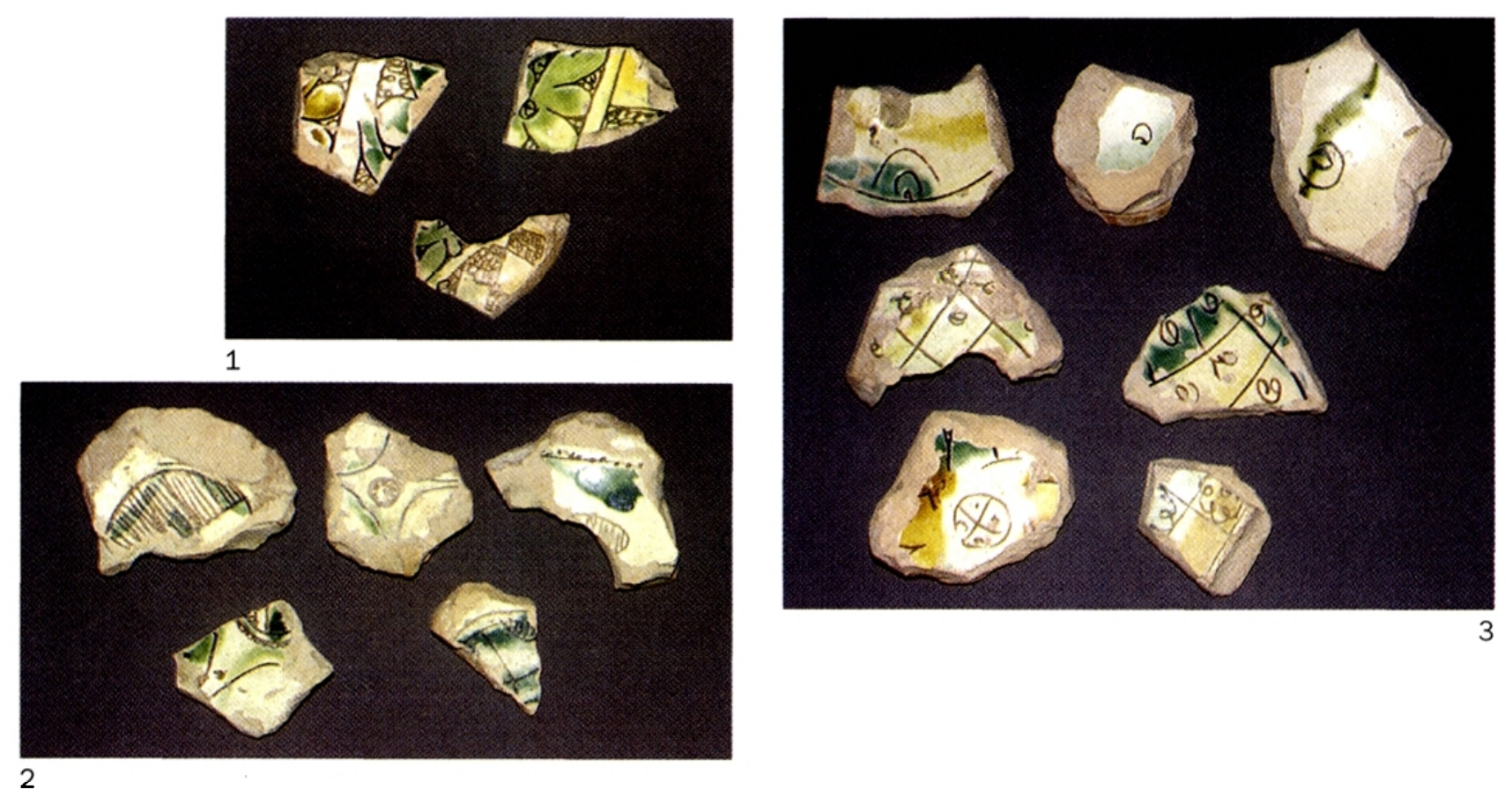

Fig. 10. Vaisselle de table de Paphos (1), de Lapithos $(2,3)$.
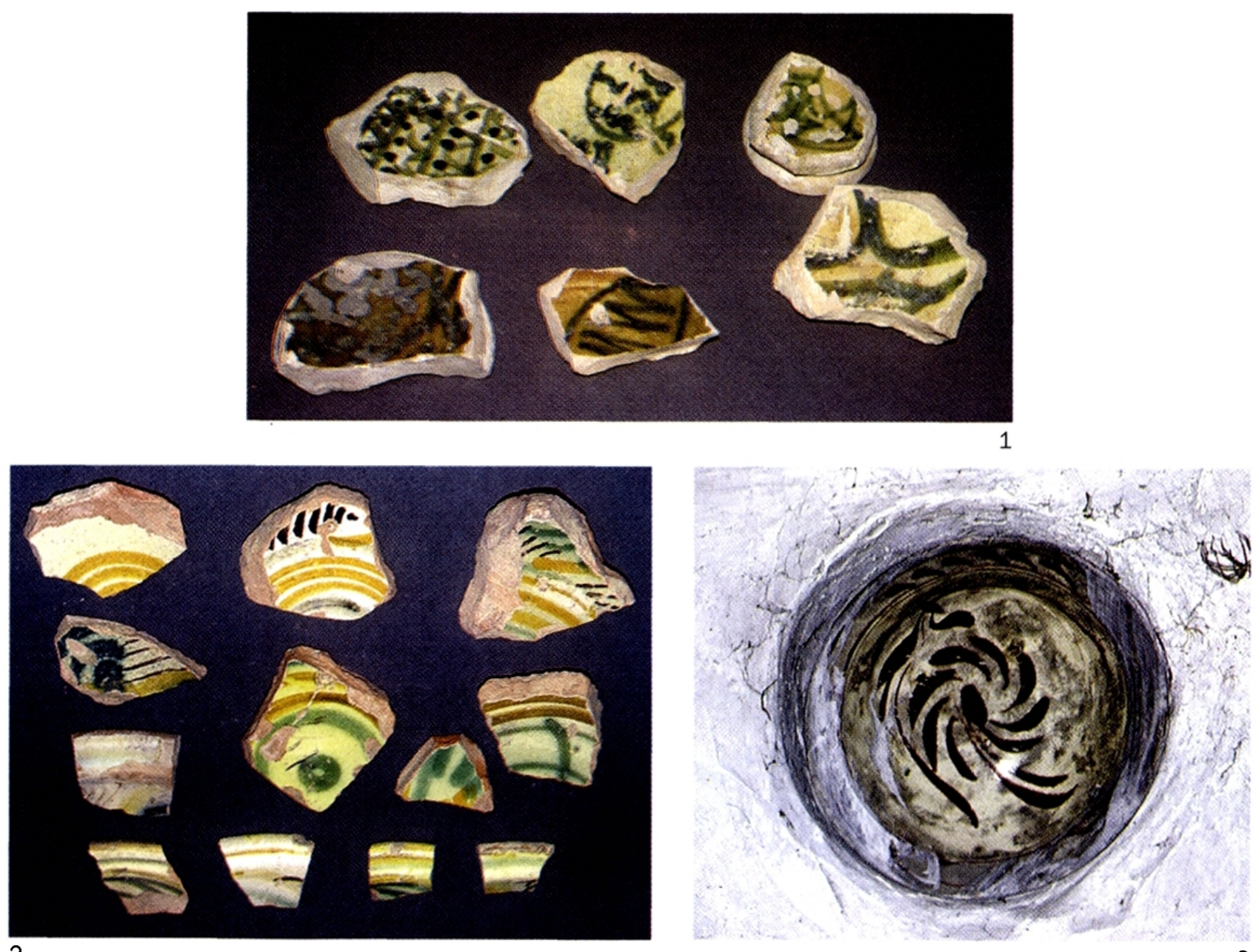

Fig. 11. Vaisselle de table de Lapithos (1); faïences de Toscane (2); céramique de Canakkale (3). 


\section{a. Importations de l'Empire ottoman}

Les productions haut de gamme des ateliers impériaux d'Iznik ne sont illustrées que par un seul tesson de très petite taille, qui peut vraisemblablement être attribué aux premières fabrications polychromes de ce centre et daté du XVIe siècle. Les fouilles menées dans la vieille ville de Famagouste ont livré un beau fragment de ce type ${ }^{52}$. De la même façon, les faïences des ateliers de Kütahya ne sont représentées que par un unique exemplaire du XVIII siècle, sans doute un fragment de brûle-parfum. Les fincan-s, ou petites tasses à café, très répandues dans tout l'Empire ottoman, sont curieusement absentes de Potamia. Les céramiques ottomanes les plus fréquemment attestées proviennent des officines de Çanakkale dans les Dardanelles, dont la production débute au milieu du XVIII siècle et se poursuit sans grand changement jusqu'à la fin du XIXe. Les coupes à marli sur pied annulaire, tournées dans une argile rouge orangé assez tendre, sont couvertes par une couche d'engobe blanc ivoire sur les deux faces et jusqu'au pied (fig. 8, $\mathrm{n}^{\text {os }} 4,5$ ). Des bouquets bleus ou bruns sont peints avec de larges coups de pinceaux au centre et bordés par une suite de petites touches sur les marlis. Ce type de vases, daté de la fin du XVIII siècle, orne la voûte de l'église d'Agios Georgios dans le village d'Agios Sozomenos (fig. 11, $\mathbf{n}^{\circ} 3$ ). Cette pratique est également attestée dans l'église de la Panagia Kanakaria à Lythrankomi ${ }^{53}$ où l'on remarque, parmi les plats emmurés dans la voûte du narthex — à la fin du XVIII siècle, comme l'indique une inscription -, des fä̈ences spirale verdi de Montelupo et des assiettes à décor ucelli e prezzemolo de Ligurie associées à quelques vases ottomans originaires de Thrace, semblables à ceux qui ont été découverts à Potamia. Il n'est en effet pas rare de trouver sur le site de larges coupes sur pied annulaire en pâte brun-rouge, à bord en crochet anguleux et décor d'engobe sous glaçure verte ou jaune, avec au centre deux cercles en léger relief (fig. 8, $\mathrm{n}^{\text {os }}$ 6-10). L'origine de ces produits qui circulent sur de longues distances n'est pas encore clairement reconnue ${ }^{54}$. Ils pourraient se rattacher aux ateliers de Didymotique, en Thrace ${ }^{55}$, dont l'activité a duré de la fin du XVII siècle jusqu'au XIXe. Quelques plats ornés d'engobes marbrés ou de simples glaçures monochromes, datant comme les précédents de la fin du XVIII siècle, ont la même origine. A Chypre, des vases de Didymotique ont été retrouvés dans la baie de Kerathidi, sur la côte occidentale de l'île $e^{56}$, et à Paphos ${ }^{57}$. Plusieurs fragments de coupelles et de formes fermées, à pâte rouge, fine et dure, couverts d'une glaçure jaune citron, se rattachent à une production ottomane représentée par une grande assiette à marli exposée au musée de Limassol mais dont l'origine n'est pas connue. Quelques fourneaux décorés de pipes à tabac ${ }^{58}$ sont trop fragmentaires pour être

52 T. MogaBgaB (supra, n. 38), p. 190, pl. XXXVI, 3.

53 A. H. S. MEGAW, E. J. W. HAWKINS, "The Church of the Panagia Kanakaria at Lythrankomi in Cyprus. Its Mosaics and Frescoes ", Dumbarton Oaks Studies 14 (1977), p. 15, fig. 21, $22,26,27$.

$54 \mathrm{~V}$. FRANÇOIS, "Byzantine ou ottomane? Une céramique peinte à l'engobe découverte en Méditerranée orientale ", Anatolia Antiqua 3 (1995), p. 203-217 ; H. AMOURIC et al., op. cit. (supra, n. 44), p. 154-157.

55 Ch. BAKIRTZIS, "Didymoteichon: un centre de céramique post-byzantine ", Balkan Studies 21 (1980), p. 147-153. 56 C. E. MorRIS, A. A. D. PEATFIELD, "Pottery from the Cyprus Underwater Survey, 1983 ", RDAC 1987, p. 200-201, fig. 1, $040,041,043$, fig. 2, 072, pl. LVIII, 8 et 9.

57 F. GIUDICE et al. (1996), loc. cit. (supra, n. 14), p. 239 240, fig. 27, no 7, tav. XXXV; id. (1994), loc. cit. (supra, n. 14), p. 256-257, pl. 16, nos 3, 4, tav. LXX.

58 Pour une large bibliographie sur le sujet, voir J. W. HAYES, op. cit. (supra, n. 49), p. 391-394, 442. 
datés précisément entre le XVII et le XIX' siècle. Enfin, un morceau de panse carénée, en pâte fine, grise, ornée de lignes géométriques et de branchages stylisés incisés, est sans doute un fragment de vase à filtre de même aspect que les gargoulettes présentées au musée de Limassol.

\section{b. Importations italiennes et provençales}

Aux XVI et XVII siècles, la circulation des produits toscans et ligures est bien reconnue dans tout le bassin méditerranéen. Nombre de navires coulés avec leur chargement en témoignent. Cependant à Potamia, les vaisselles pisanes du XVII siècle ne sont attestées que par deux fragments en pâte rouge brique aux couleurs vives, vernissées sur engobe et aux décors incisés. Cette céramique graffita tarda est cependant représentée sur l'île par une coupe creuse décorée d'un oillet" déposée dans une tombe près de l'église de la Chrysanayiotissa; et par d'autres exemplaires conservés dans les vitrines du musée de Limassol. Onze fragments de coupes en faïence polychrome toscanes à décor de spirale verdi pourraient être attribués aux officines pisanes ou à un autre centre toscan du XVIII siècle mais l'argile rouge exclut une origine florentine (fig. 11, $\mathbf{n}^{\circ}$ 2). L'église de la Panayia Kanakaria à Lythrankomi en conserve un exemplaire encore en place dans la voûte du narthex et associé à des faïences ligures peintes en bleu et jaune $a$ prezzemolo et a uccelli ${ }^{60}$. L'autre production qui marque l'apothéose de la Ligurie au XVIII siècle repose sur deux gammes d'articles, les terrailles noires d'Albisola et les blanches dites de Rome mais néanmoins originaires de la Rivière de Gênes. Une vingtaine de fragments d'assiettes en pâte rouge recouverte de glaçure plombifere brune peinte en noir, et une dizaine de fragments d'assiettes en pâte blanche émaillée, illustrent cette circulation à Chypre. La présence d'une vingtaine de tessons de marmites de Vallauris issues des célèbres officines des Alpes-Maritimes qui sont, au XVIII siècle, au premier rang dans le secteur des vaisselles culinaires, est plus étonnante. Les cols à lèvre carrée en pâte kaolinitique glaçurée en brun-jaune ou miel munis de deux anses verticales sont accompagnés de quelques épais fragments de panses de jarres de Biot à glaçure jaune clair. Ces derniers ateliers, spécialisés dans la production de gros contenants pour le stockage et le transport, expédiaient sans doute leurs produits par les mêmes voies que les ateliers voisins ligures. L'universalité des productions de Provence orientale est illustrée aussi dans les jardins du village de Dhali, où subsistent encore des jarres. Enfin quelques vaisselles engobées de l'arrière-pays marseillais complètent le répertoire issu de la Méditerranée occidentale ${ }^{61}$. Toutes ces productions du XVIII siècle, - les assiettes de Ligurie, les marmites et les jarres de VallaurisBiot et les coupes de Marseille - furent largement distribuées non seulement dans le bassin méditerranéen mais aussi dans les îles françaises d'Amérique, en Guyane et au Canada.

59 J. DU PLAT TAYLOR, loc. cit. (supra, n. 5), fig. 20, forme 5 ,

p. 67 ; H. AMOURIC et al., op. cit. (supra, n. 44), p. 79-86.

60 A. H. S. MEGAW, E. J. W. HAWKINS, loc. cit. (supra, n. 53),

fig. 26, 27 ; H. AMOURIC et al., loc. cit. (supra, n. 44), p. 121-

127.

61 ibid., p. 128-142. 
Les céramiques recueillies au cours de la prospection de la région de Potamia, malgré leur état très fragmentaire, ont permis de livrer de nouvelles informations à l'échelle du site mais aussi à l'échelle de l'île pour les époques franque et ottomane. Parmi elles, il faut d'abord retenir l'existence, à Potamia, d'une fabrication de céramique à pâte calcaire ainsi qu'une production régionale de vaisselle culinaire à pâte rouge, glaçurée. Il convient ensuite d'insister sur la nécessité de présenter autant que faire se peut les vases d'époque ottomane encore négligés dans les publications. Les études ponctuelles et répétées de ce matériel devraient permettre à terme d'aboutir à une typologie du vaisselier chypriote ottoman. Enfin, il nous a paru important de distinguer finement les importations médiévales et modernes venues d'Espagne, d'Italie et du Sud de la France et qui, jusqu'à maintenant, étaient passées un peu inaperçues. Ces nouvelles données sur la production et la consommation de céramique à Chypre devront être nourries par les découvertes faites dans les futures fouilles de Potamia-Agios Sozomenos ${ }^{62}$.

62 L'examen du matériel s'est poursuivi en janvier 2002. II confirme le faciès céramologique précédemment établi. Cependant, on note la présence de quelques types nouveaux importés de Méditerranée occidentale, tels que de la céramique incisée, "sgraffito archaïque * ligure du XIIIe s., de la céra- mique marmorizzate de Pise du XVIIe s.; de Méditerranée orientale, de la céramique Fine Sgraffito Ware, Green and Brown Painted Sgraffito Ware du XII' s., ainsi qu'une fincan-s de Kutahya à décor moulé polychrome du Xville $\mathbf{s}$. 